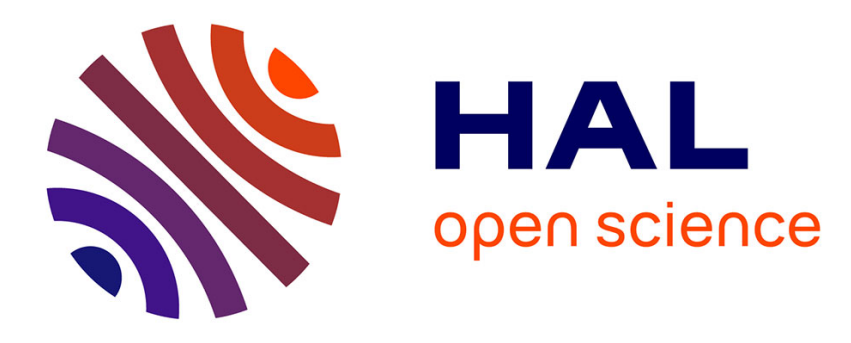

\title{
Spin-orbit effects in ferroelectric PbTiO3 under tensile strain
}

J. Gosteau, Rémi Arras, P. Chen, H. Zhao, Charles Paillard, Laurent Bellaiche

\section{To cite this version:}

J. Gosteau, Rémi Arras, P. Chen, H. Zhao, Charles Paillard, et al.. Spin-orbit effects in ferroelectric PbTiO3 under tensile strain. Physical Review B, 2021, 103 (2), pp.024416. 10.1103/PhysRevB.103.024416 . hal-03295044

\section{HAL Id: hal-03295044 \\ https://hal.science/hal-03295044}

Submitted on 11 Oct 2021

HAL is a multi-disciplinary open access archive for the deposit and dissemination of scientific research documents, whether they are published or not. The documents may come from teaching and research institutions in France or abroad, or from public or private research centers.
L'archive ouverte pluridisciplinaire HAL, est destinée au dépôt et à la diffusion de documents scientifiques de niveau recherche, publiés ou non, émanant des établissements d'enseignement et de recherche français ou étrangers, des laboratoires publics ou privés. 


\title{
Spin-orbit effects in ferroelectric $\mathrm{PbTiO}_{3}$ under tensile strain
}

\author{
J. Gosteau and R. Arras $\odot^{*}$ \\ CEMES, Université de Toulouse, CNRS, UPS, 29 Rue Jeanne Marvig, F-31055, Toulouse, France \\ P. Chen $\odot$ and H. J. Zhao $\odot$ \\ Physics Department and Institute for Nanoscience and Engineering University of Arkansas, Fayetteville, Arkansas 72701, USA \\ C. Paillard $\odot$ \\ Laboratoire SPMS, CentraleSupélec/CNRS UMR8580, Université Paris-Saclay, 8-10 Rue Joliot-Curie, 91190 Gif-sur-Yvette, France \\ L. Bellaiche \\ Physics Department and Institute for Nanoscience and Engineering University of Arkansas, Fayetteville, Arkansas 72701, USA
}

(Received 22 October 2020; accepted 22 December 2020; published 12 January 2021)

\begin{abstract}
We report a study based on first-principles calculations complemented by a tight-binding approach and a $\boldsymbol{k} \cdot \boldsymbol{p}$ model of the spin-orbit effects in epitaxially strained $\mathrm{PbTiO}_{3}$. The imposed biaxial tensile strain allows us to rotate the electrical polarization of $\mathrm{PbTiO}_{3}$ from out-of-plane to in-plane directions through structural phase transitions between a tetragonal, monoclinic, and orthorhombic phase. It is found that $\mathrm{Ti} d$-state conduction bands exhibit two strong Rashba-like spin-splitting parameters of (1) $0.39 \mathrm{eV} \AA$ at a tensile strain $\eta=1.55 \%$ due to a polarization rotation in the monoclinic phase and (2) $1.08 \mathrm{eV} \AA$ under large tensile strain of $3.8 \%$ due to band anticrossing in the orthorhombic phase. Remarkably, we also identified the presence of a quasipersistent spin texture for the band with a $d_{y z-z x}$ character in the orthorhombic phase. We then conclude that using strain could be an interesting way to tune the spin-orbit effects in ferroelectric materials with technological interests.
\end{abstract}

DOI: 10.1103/PhysRevB.103.024416

\section{INTRODUCTION}

Understanding spin-orbit effects and manipulating the spin degree of freedom in materials is considered nowadays as one of the most promising ways to develop a part of the next generation of low-consumption-operating electronic devices [1-6]. In 1955, spin-orbit effects have been highlighted in nonmagnetic semiconductors lacking spatial inversion symmetry [7]: Linked with the bulk inversion asymmetry (BIA), the now called Dresselhaus effect relates the variation of the energy difference $\Delta E_{ \pm}=E_{+}-E_{-}$between the two nondegenerate bands of opposite spin projection ("+" or "-") to third-order polynomials in the wave vector $\boldsymbol{k}$. This is the result of the addition of a perturbative term to the Hamiltonian, which can be approximated to the linear term $\sim \alpha_{\mathrm{D}}\left(k_{x} \sigma_{x}-k_{y} \sigma_{y}\right)$ in the limit of quasi-two-dimensional (2D) systems [8]. In this equation, $\alpha_{\mathrm{D}}$ is the Dresselhaus parameter which characterizes the strength of the spin-orbit effect; $\sigma_{i}$ are the Pauli matrices and $k_{i}$ the components of the wave vector $k$, with $i=x, y, z$ the Cartesian space coordinates. In 1984, another spin-orbit term, with the form $\alpha_{\mathrm{R}}\left(\sigma_{y} k_{x}-\sigma_{x} k_{y}\right)$, was proposed to describe the linear spin splitting observed in 2D systems [9] (surfaces, interfaces, or quantum wells): The Rashba effect, characterized by the spin-orbit parameter $\alpha_{\mathrm{R}}$, is now more commonly associated to a surface inversion asymmetry (SIA); it is however a general effect which can appear in any structure lacking inversion symmetry, with an "at-least-threefold"

*remi.arras@cemes.fr symmetry axis and an invariant vector along this axis (such as a polar vector) [9]. This effect was subsequently evidenced and studied in several bulk or heterostructures based on semiconductors $[10,11]$, heavy metals $[12,13]$, or transition-metal oxides [14-16]. In addition to the Dresselhaus and Rashba effects, recent and more systematic investigations have shown that many corrective terms can be proposed to describe the spin-orbit interaction, the form of which will depend on the space group of the considered material (or that of the surface/interface orientation) $[17,18]$. Depending on the considered bands, higher-than-linear terms in vector $\boldsymbol{k}$ may be necessary to describe accurately the spin splittings and spin textures.

Besides its interest at a fundamental level, the Rashba spinorbit effect has recently motivated lots of research effort due to its possibility to be used in innovative spintronic devices with low energy consumption. The Rashba effect can indeed be viewed as a functional property since its magnitude can be tuned by applying an electric field. This effect can also play a role in transport processes, enabling in particular the creation or detection of spin current through the spin Hall effect $[19,20]$ or the (inverse) spin-galvanic effect, also known as 2D Rashba-Edelstein effect [21-24]. The spin-to-charge conversion using spin-orbit effects is very promising for the development of current spin-orbitronic applications and devices. Thanks to the so-called spin-orbit torque, this property could allow us to manipulate efficiently the magnetization in an adjacent magnetic electrode in magnetic random access memories (MRAM) [6]. It could also be used in new concepts of nonvolatile devices such as the magnetoelectric 
spin-orbit (MESO) device [2], proposed to challenge current complementary-metal-oxide-semiconductor (CMOS) technology with lower operating costs or in the famous spin field-effect transistor (spin-FET), in which the rotation of the spins of the electrons moving in the channel can be controlled by the applied voltage [1]. Because of their advantages to create, manipulate, or detect the spin currents, it would be logical to try to improve systematically the strength of the spin-orbit effects, but one has to keep in mind that they can also be detrimental to spin transport due to the spin dephasing they can induce [25]. Such phenomena could be avoided by using materials possessing persistent spin textures (PST) [18], which can arise from a compensation of the Rashba and Dresselhaus term $\left(\alpha_{R}=\alpha_{D}\right)$ or be enforced directly by symmetry for some crystal structures [16,26,27].

In 2013, the Rashba effect has been predicted theoretically and then confirmed experimentally in the ferroelectric Rashba semiconductor (FERSC) GeTe, with a Rashba value $\alpha_{\mathrm{R}}=4.9$ $\mathrm{eV} \AA$ [28-30]. This spin-orbit effect appears to have a strong interplay with the electric polarization, leading to spin textures reversible by manipulating the electric polarization. This behavior is very promising to design innovative devices based on nonvolatile magnetoelectric states. The narrow band gap of GeTe appears, however, to be detrimental to retain the electric polarization and further to control the Rashba-induced spin textures [31]. This seminal work has motivated several new investigations to get a better understanding on the spin-orbit effect in this class of materials and to potentially find ferroelectrics more suitable for applications [5,15,16,32-36].

Different studies also proposed to analyze the variation of the Rashba parameter as a function of a biaxial strain [15,3739]. In their theoretical work, Yamaguchi and Ishii [38] explored the effect of a tensile strain on the spin-orbit coupling on the well-known $\mathrm{LaAlO}_{3} / \mathrm{SrTiO}_{3}(001)$ interface: They observed an increase of the Rashba effect up to $5 \%$ for a tensile strain of $7 \%$ with the emergence of an in-plane polarization giving rise to a persistent spin helix. In 2016, Tao and Wang [15] studied the strain-tunable ferroelectricity of nominally paraelectric $\mathrm{KTaO}_{3}$. In particular, they found that the out-of-plane electric polarization induced by a compressive in-plane strain can be switched in-plane with a tensile strain. Such switching leads to a change in the spin-texture orientation, since the Rashba lifting of spin degeneracy only occurs in directions perpendicular to the electric polarization. These two studies thus show that other crystallographic phases, with a transition of the electric polarization from out-of-plane to in-plane, can be stabilized by applying a tensile strain, thus adding a supplementary degree of freedom to manipulate the spin textures. The possibility to get a PST for an in-plane polarization is moreover very promising. It is thus interesting to mention the work of Djani et al. [16], who investigated the spin-orbit effect in various phases (in particular $P 4 \mathrm{~mm}$ and $A m m 2$ ) of the ferroelectric perovskite $\mathrm{WO}_{3}$ and the aurivilius $\mathrm{Bi}_{2} \mathrm{WO}_{6}$. They proposed that in perovskite structure an orbital character perpendicular to the polarization leads to a weak Rashba parameter, while a band orbital with a component collinear to the polarization yields a stronger Rashba value. They also found a persistent spin texture for the $P 2_{1} a b$ phase of $\mathrm{Bi}_{2} \mathrm{WO}_{6}$, for which the electric polarization is in-plane.
From the above review, we can conclude that the study of the spin-orbit effects in different crystallographic phases of $\mathrm{PbTiO}_{3}$ (PTO) could be very promising. This material is indeed a textbook case in the ferroelectrics family and it is now used for many applications, either in its pure form or in alloyed compositions [40-43]. PTO holds a measured electric polarization of about $75 \mu \mathrm{Ccm}^{-2}$ [44] and an experimental band gap of $3.4 \mathrm{eV}$ [45]. From numerical studies, the electric polarization in PTO is expected to be robust to electron doping [46-48], which would be required to address the spin-splitted conduction bands, and it could persist in thin films, in the vicinity of (001)-oriented surfaces or interfaces [49-54]. In addition, this compound possesses a heavy metal, which can potentially reinforce the spin-orbit effects. It also has a simple perovskite structure and $\mathrm{Ti}^{4+}$ cations located in the octahedral atomic sites, which can be linked to a band structure displaying similar properties to $\mathrm{SrTiO}_{3}$-based heterostructures $[55,56]$.

Recently, we conducted a numerical study focused on the spin-orbit effects in the ferroelectric and tetragonal phase $(P 4 \mathrm{~mm})$ of PTO [35]. Our results showed that, depending on the symmetry of the lowest conduction band (Ti- $d_{x y}$ or $\mathrm{Pb}-p_{z}$ ), the spin splitting could display a variation dominated by linear or cubic terms in $\boldsymbol{k}$. If we consider (i) the calculated equilibrium in-plane lattice parameter of PTO to be $a_{0}=3.881 \AA$ and (ii) $a$ to be the in-plane lattice parameter resulting from epitaxial growth, we can then define the applied biaxial tensile strain via $\eta=\left(a-a_{0}\right) / a_{0}$. Note that it has been found that applying a compressive strain $(\eta<0 \%)$ up to $-5 \%$ only increases the tetragonality characterized by the $c / a$ ratio (where $c$ is the out-of-plane lattice parameter) and the electric polarization, but does not change the crystallographic phase $[57,58]$. This quasilinear variation of the structural parameters is also accompanied by a linear decrease (increase) of the linear(cubic) terms of the $p_{z}\left(d_{x y}\right)$ bands, in correlation with a change of the band character. If an in-plane compressive strain increases the out-of-plane electric polarization and the tetragonality of PTO [35], growing epitaxially PTO on substrates with larger in-plane lattice parameters would allow us to modify the crystallographic structure and induce a rotation of the electric polarization [57-60].

We propose in the current study to complete our previous work and to analyze the spin-orbit effects in PTO under tensile strain by probing their variations in three crystallographic phases with a different orientation of the electric polarization. We will show that the spin splittings and spin textures can be tuned as a function of the strain because of a modification of the electric-polarization magnitude or angle. In particular, we found that a quasipersistent spin texture can be achieved in the Amm 2 phase. To do so, we will first discuss the changes induced in the atomic structure as a function of the applied in-plane strain. Secondly, we will present the evolution of the spin-splitting values calculated from first principles and we will finally give a tight-binding and a $\boldsymbol{k} \cdot \boldsymbol{p}$ model based on symmetry invariants to propose some explanations.

\section{COMPUTATIONAL DETAILS}

We performed density functional theory (DFT) calculations by using the Vienna ab initio simulation package 
TABLE I. Structural parameters of $\mathrm{PbTiO}_{3}$ for three different strain states: $a$ and $c$ correspond, respectively, to the fixed in-plane and calculated out-of-plane lattice parameters; $\beta$ is the angle between $a$ and $c$ axes. $(x, y, z)$ are the reduced coordinates [in units of $(a, a, c)$ ] of the $\mathrm{Ti}, \mathrm{O}_{\|}$, and $\mathrm{O}_{\perp}$ atoms, with the $\mathrm{Pb}$ atom set as the origin $(0,0,0)$ of the basis. $\mathrm{O}_{\|}$is one of the two oxygen atoms lying in the $\mathrm{TiO} \mathrm{O}_{2}(001)$ atomic layer, parallel to the in-plane strain, and $\mathrm{O}_{\perp}$ is the oxygen atom in the $\mathrm{PbO}(001)$ plane.

\begin{tabular}{|c|c|c|c|c|c|c|c|c|c|c|c|c|c|}
\hline \multirow[b]{3}{*}{ Phase } & \multirow[b]{3}{*}{$\eta(\%)$} & \multirow[b]{3}{*}{$a(\AA)$} & \multirow[b]{3}{*}{$c(\AA)$} & \multirow[b]{3}{*}{$\beta\left(^{\circ}\right)$} & \multicolumn{9}{|c|}{ Reduced coordinates } \\
\hline & & & & & \multicolumn{3}{|c|}{$\mathrm{Ti}$} & \multicolumn{3}{|c|}{$\mathrm{O}_{\|}$} & \multicolumn{3}{|c|}{$\mathrm{O}_{\perp}$} \\
\hline & & & & & $x$ & $y$ & $z$ & $x$ & $y$ & $z$ & $x$ & $y$ & $z$ \\
\hline$P 4 m m$ & 0.00 & 3.881 & 4.156 & 90.00 & 0.50 & 0.50 & 0.54 & 0.50 & 0.00 & 0.62 & 0.50 & 0.50 & 0.11 \\
\hline $\mathrm{Cm}$ & 1.87 & 3.953 & 3.944 & 90.48 & 0.52 & 0.48 & 0.52 & 0.55 & -0.05 & 0.56 & 0.55 & 0.45 & 0.05 \\
\hline Amm2 & 3.60 & 4.021 & 3.862 & 90.00 & 0.52 & 0.48 & 0.50 & 0.57 & -0.06 & 0.50 & 0.41 & 0.43 & 0.00 \\
\hline
\end{tabular}

(VASP) [61,62] with projector-augmented wave PAW pseudopotentials [63]; the following electrons were treated as valence states: $\mathrm{Pb}\left(6 s^{2} 5 d^{10} 6 p 2\right)$, $\mathrm{Ti}\left(3 s^{2} 3 p^{6} 4 s^{2} 3 d^{2}\right)$, and $\mathrm{O}\left(2 s^{2} 2 p^{4}\right)$. A cutoff energy of $750 \mathrm{eV}$ and the generalized gradient approximation for the exchange-correlation energy with the Perdew-Burke-Ernzerhof version revised for solids (GGA-PBESol) [64] were used. The first Brillouin zone was sampled by a $10 \times 10 \times 10$ Monkhorst-Pack grid [65].

All the band energies are further given relatively to the Fermi energy $E_{\mathrm{F}}$, which is arbitrarily set to the valence band maximum (VBM). The spin-orbit interaction, as implemented in VASP [66], has been added self-consistently for the DFT calculations. The setup of the $\boldsymbol{k} \cdot \boldsymbol{p}$ Hamiltonians has been made using the resources from the Bilbao crystallographic server [67,68] and Refs. [11,69-71]. The different spin-orbit parameters of the $\boldsymbol{k} \cdot \boldsymbol{p}$ model have been calculated by minimizing the sum of least squares given by:

$$
\chi^{2}=\sum_{\boldsymbol{k}}\left[\operatorname{det}\left(H(\boldsymbol{k})-E_{ \pm}(\boldsymbol{k})\right)\right]^{2},
$$

where $H$ is the two-band Hamiltonian representing the lift of degeneracy undergone by the two spin states and caused by the spin-orbit interaction. The fits have been performed on the DFT band structures calculated around the $\Gamma$ point, with a maximum magnitude of the $\boldsymbol{k}$ wave vectors of 0.10 $\AA^{-1}$ sampled with at least $40 \boldsymbol{k}$ vectors. We also used a tightbinding (TB) model built from the projections onto maximally localized Wannier functions of the conduction bands $(\mathrm{Pb}-p$ and Ti- $d$ bands) including spin-orbit coupling; the projections have been performed using the Wannier90 code [72].

Our calculations were made using a five-atoms perovskite unit cell; $\sqrt{2} \times \sqrt{2} \times 2$ supercells containing 20 atoms were also used to check the possible existence of other crystallographic phases as will be discussed in more details in the next section. The space group of the different phases has been identified using the FINDSYM program $[73,74]$ with a tolerance of $10^{-2} \AA$. The epitaxial in-plane strain $\eta$ was modeled by fixing the in-plane lattice parameter $a=b$ for different space groups. The tensile biaxial strain $\eta$ has been applied from a range of 0 to $5 \%$. In the following, the Cartesian axes are always defined to coincide with the axes of the $P 4 \mathrm{~mm}$ phase: The $x[100]$ and $y[010]$ axes are the directions of the biaxial strain. The $z$ axis is defined as the perpendicular to $x$ and $y$ ([001] direction of the $P 4 m m$ phase). To simplify the comparison of the band structures, we also chose to keep for every structure the same notations for the high-symmetry points as for the $P 4 \mathrm{~mm}$ phase. The equivalence between our notations and the standard notations provided by the Bilbao Crystallographic Server $[67,68]$ is given in Table II of Appendix A. The electric polarization has been calculated using the Berry phase method [75].

\section{STRUCTURAL PROPERTIES}

Before discussing the electronic structure and the spinorbit effects in the next sections, we will describe here the influence of the tensile biaxial strain $\eta$ on the atomic structure. The calculated equilibrium crystallographic phase $(\eta=$ $0 \%)$ of PTO corresponds to a tetragonal structure $\left(a_{0}=b_{0}=\right.$ $3.881 \AA$ and $c_{0}=4.156 \AA$ ) with the space group $P 4 m m\left(n^{\circ} 99\right)$ and an electric polarization of $91 \mu \mathrm{C} \mathrm{cm}^{-2}$, oriented along the $z$ [001] axis. These theoretical lattice parameters are in agreement with the experimental values of $a_{\exp }=3.89-3.90$ $\AA$ and $c_{\text {exp }}=4.14-4.16 \AA[76,77]$. When varying $\eta$ from 0 to $5 \%$, we calculated a decrease of the ratio between the out-of-plane and in-plane lattice parameters $(c / a)$ from 1.07 to 0.94 , which will have different consequences on the atomic structure and in particular induce a change of the orientation of the electric polarization regarding the strain directions.

Figure 1 shows the variation of the total energy $E_{0}$ calculated for different values of $\eta$ and the induced changes in the electric polarization orientation and magnitude. From Fig. 1(a), we can see that the $P 4 m m$ phase remains stable up to $\eta=1.3 \%$, albeit with a smaller electric polarization [see Fig. 1(c)] of $P(\eta=1.3 \%)=81 \mu \mathrm{C} \mathrm{cm}^{-2}$. When increasing $\eta$ above $1.3 \%$, PTO adopts a monoclinic structure with a space group $\mathrm{Cm}\left(\mathrm{n}^{\circ} 8\right)$. Within this phase, the polarization continuously rotates in a (110) plane, from its out-of-plane direction to the [1 $\overline{1} 0]$ in-plane direction. Above $\eta=2.5 \%$,

TABLE II. Equivalence in the notations between the highsymmetry points of the $P 4 \mathrm{~mm}, \mathrm{Cm}$, and Amm2 crystallographic phases according to Refs. $[67,68]$. The point coordinates are given in units of the reciprocal lattice parameters $a^{*}, b^{*}$, and $c^{*}$.

\begin{tabular}{lccc}
\hline \hline Coordinates & $P 4 m m$ & $C m$ & $A m m 2$ \\
\hline$(0,0,0)$ & $\Gamma$ & $\Gamma$ & $\Gamma$ \\
$(0,0.5,0)$ & $\mathrm{X}$ & $\mathrm{V} 2$ & $\mathrm{~S}$ \\
$(0.5,0.5,0)$ & $\mathrm{M}$ & $\mathrm{Y}$ & $\Sigma 0$ \\
$(0,0,0.5)$ & $\mathrm{Z}$ & $\mathrm{A}$ & $\mathrm{Z}$ \\
$(0,0.5,0.5)$ & $\mathrm{R}$ & $\mathrm{L} 2$ & $\mathrm{R}$ \\
$(0.5,0.5,0.5)$ & $\mathrm{A}$ & $\mathrm{I} 2$ & $\mathrm{~A} 0$ \\
\hline \hline
\end{tabular}


(a)

(b)
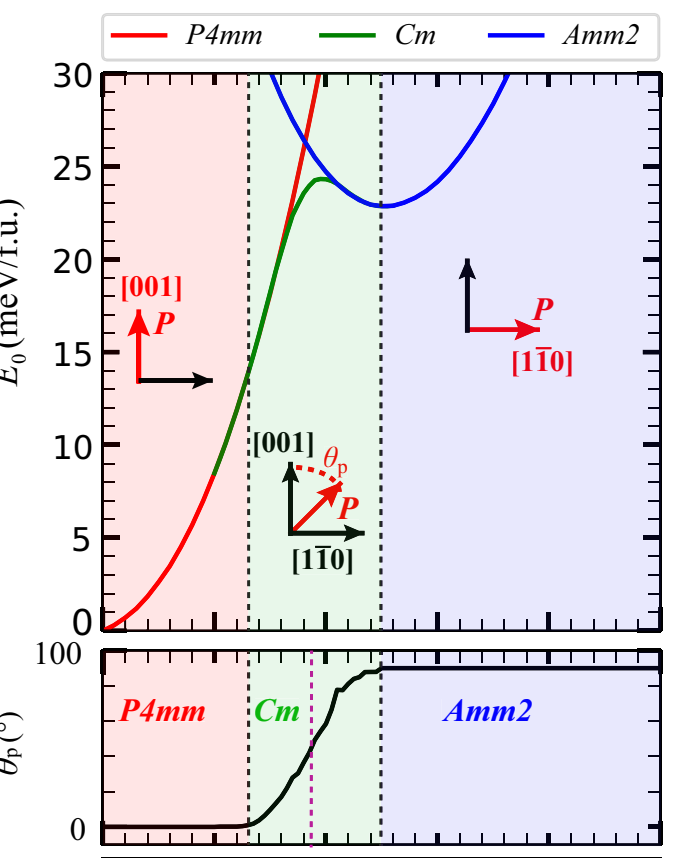

(c)

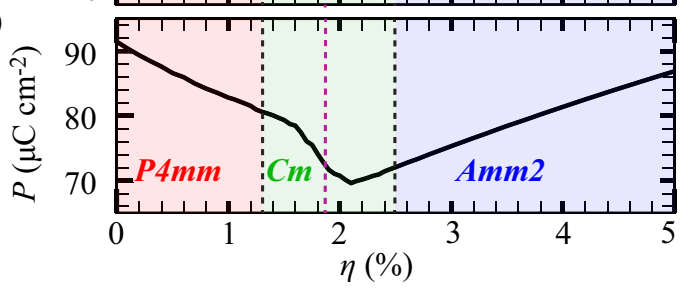

FIG. 1. (a) Total energies $E_{0}$ calculated for the tetragonal $(\mathrm{P} 4 \mathrm{~mm})$, monoclinic $(\mathrm{Cm})$, and orthorhombic $(\mathrm{Amm} 2)$ phases of $\mathrm{PbTiO}_{3}$ as a function of the in-plane strain $\eta$. The red arrows are a sketch of the polarization orientation in the basis $\{[1 \overline{1} 0]$, [001]\}. (b) Angle $\theta_{p}$ between the electric polarization $\boldsymbol{P}$ and the $z[001]$ axis and (c) magnitude of the polarization calculated in $\mathrm{PbTiO}_{3}$ for the most stable crystallographic phases depending on $\eta$. The dotted purple lines indicate the value of strain $\eta$ for which the electric polarization vector is nearly aligned with the [111] direction.

when the polarization is in-plane, an orthorhombic structure of space group Amm2 ( $\left.{ }^{\circ} 38\right)$ is stabilized. Following the variation of the in-plane parameters, the magnitude of the electric polarization will start again to increase, from $\simeq 72 \mu \mathrm{C} \mathrm{cm}^{-2}$ to $\simeq 87 \mu \mathrm{C} \mathrm{cm}^{-2}$ when increasing the strain up to $\eta=5 \%$. Finally, Table I summarizes the lattice parameters for three different strain states $(\eta=0,1.87,3.6 \%)$, corresponding each one to one of the above-mentioned crystallographic phases, respectively, with the $P 4 \mathrm{~mm}, \mathrm{Cm}$, or Amm 2 space group; we also provide the reduced coordinates for these structures.

In addition to the above description of the structural transformations undergone by PTO, some remarks can be made:

(1) Firstly, we can distinguish particular values of the strain of approximately $\eta=1.86-1.87 \%$ for which the inplane and out-of-plane parameters are almost equal $(c / a \simeq 1)$ and the electric polarization $\boldsymbol{P}$ forms an angle of $\theta_{p} \simeq 55^{\circ}$ with the $z[001]$ axis, i.e., $\boldsymbol{P}$ is approximately in the [1 11 ] direction. In this range of $\eta$, the structure is close to the rhombohedral space group $R 3 m\left(\mathrm{n}^{\circ} 160\right)$.
(2) Secondly, the monoclinic $\mathrm{Cm}$ phase corresponds to a rotation of the electric polarization in a (110) plane. Another slightly less stable monoclinic phase with the space group $\mathrm{Pm}$ $\left(\mathrm{n}^{\circ} 6\right)$ and corresponding to a rotation in a (100) plane has also been predicted from first-principles calculations [60].

(3) The difference between the total energy of the $\mathrm{Cm}$ phase and the $P 4 \mathrm{~mm}$ and $\mathrm{Amm} 2$ phases is maximum near $\eta=1.8 \%$, with a value of $2.38 \mathrm{meV} /$ formula unit (f.u.). Because of these small energy differences, it can be expected that different phases coexist in real samples.

(4) The last point is that, in Refs. [58,60], the authors found that the most stable orthorhombic phase corresponds to the $\operatorname{Ima} 2\left(\mathrm{n}^{\circ} 46\right)$ space group, instead of the Amm2 one. Using a 20-atom cell, we found that these two structures have a total energy almost equal (within $\sim 10^{-4} \mathrm{eV} /$ f.u.) in the full range of strain we considered. The difference between these two phases is related to slightly rotated oxygen octahedra in the Ima 2 structure, which have been calculated with angles inferior to $0.3^{\circ}$. For the sake of simplicity, we chose to neglect this distortion and to focus our following discussion only on the Amm 2 structure, which can be described by a simpler five-atom cell. Because we focused our study on the electronic structure and the related spin-orbit effects around the highsymmetry point $\Gamma$, the results presented in the following are the same for both phases, Amm2 and Ima2.

In summary, our calculations confirm that applying a biaxial in-plane tensile strain could allow us to stabilize other crystallographic phases. Experimentally, the growth of PTO films on substrates with higher lattice parameters has also already been demonstrated. We can for example mention the work of Catalan et al. [78], who reported the epitaxial growth of PTO on $\mathrm{DyScO}_{3}$ substrates with orthorhombic or pseudocubic structures. In the former case, the deposited PTO films adopted also an orthorhombic structure, while in the latter case, the author reported a coherence between the lattice parameter of the 5-nm-thick PTO films and the substrate, corresponding to an induced in-plane tensile strain of approximately $1.5 \%$. For these films, a rotation of the electric polarization from its out-of-plane direction is measured, leading to a monoclinic phase. The epitaxial growth of 90nm-thick (100)PTO films on $(001) \mathrm{KTaO}_{3}$ has been lately reported [79], corresponding to an averaged increase of the in-plane lattice parameters of $2.5 \%$; these films possessed $90^{\circ}$-domain structures with reduced in-plane electric polarizations and orthorhombiclike structures with different $a$ [100] and $b[010]$ lattice parameters. All these experimental studies show the difficulties of growing strained PTO thin films, but they also demonstrate the feasibility and their measurements are in line with our calculated structures. In addition, we can consider that it could be also possible to obtain different crystallographic phases by using $\mathrm{PbTiO}_{3}$-derived compounds with varying compositions [80-82] or by taking benefits from the emergence of flexoelectricity in some thin films [83].

\section{ELECTRONIC PROPERTIES}

In this section we will describe the electronic structure of the three different crystallographic phases which can be stabilized in PTO by varying the in-plane strain $\eta$. Figure 2 compares the band structures for these phases. 

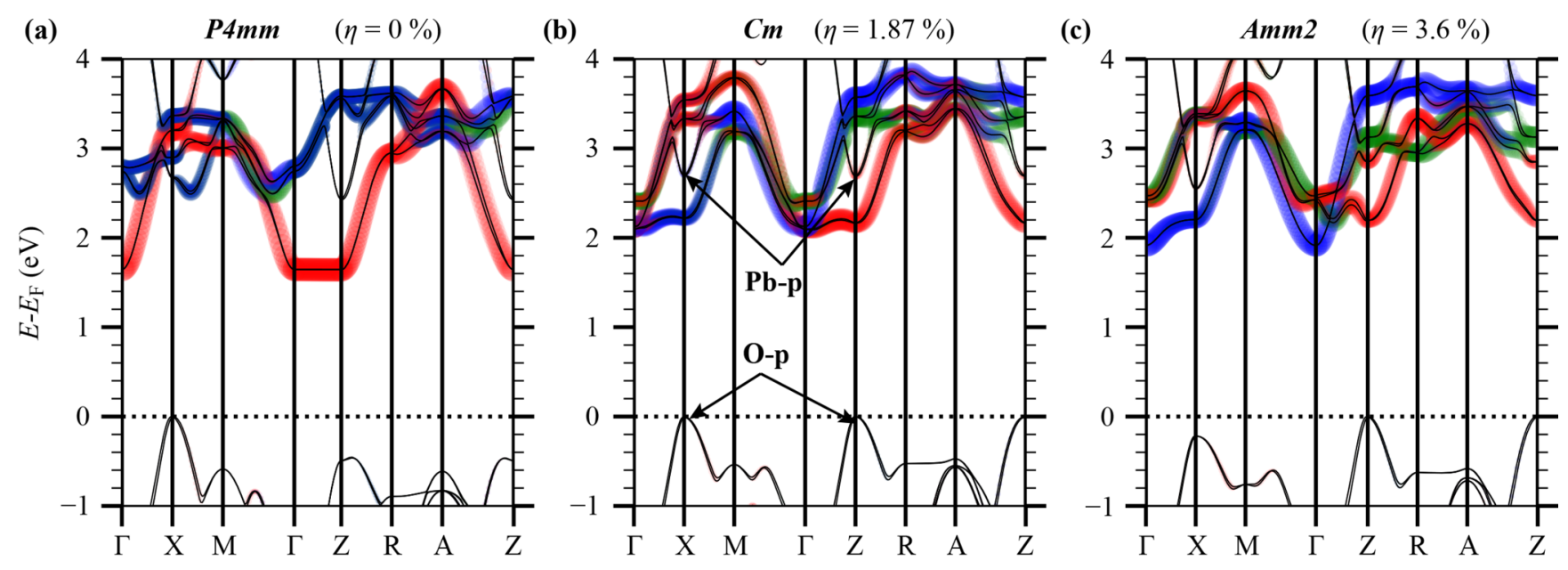

Ti: $\quad \mathrm{d}_{x y} \quad ; \quad \mathrm{d}_{y z+z x} ; \mathrm{d}_{y z-z x}$

FIG. 2. Band structures associated with the atomic structures detailed in Table I: (a) the $P 4 m m$ structure calculated for an in-plane strain of $\eta=0 \%$, (b) the $C m$ phase at $\eta=1.87 \%$, and (c) the Amm2 phase at $\eta=3.6 \%$. The Ti(d)-orbital contributions are given in color.

Considering first the equilibrium $P 4 m m$ structure at $\eta=$ $0 \%$, we calculated an indirect band gap with a width of 1.65 $\mathrm{eV}$ : The VBM is located at the $\mathrm{X}$ point and has a mixed $\mathrm{Pb}-s$ and $\mathrm{O}-p$ character, while the conduction band minimum (CBM) is at the $\mathrm{Z}$ point, with a Ti- $d_{x y}$ character and an energy close to its value at the $\Gamma$ point. The detailed analysis of the visible spin splitting present around the $\mathrm{Z}$ point for the $d_{x y}$ conduction bands of the Ti atoms and the $p_{z}$ band of $\mathrm{Pb}$ atoms was the main object of our previous study [35]. In the following, for an easier comparison between the different crystallographic phases, we will mostly focus our discussion on the spin splitting which can be observed around the $\Gamma$ point for the three lowest conduction bands.

From $\eta=1.3 \%$, when PTO adopts the monoclinic $\mathrm{Cm}$ phase, the valence bands at the $\mathrm{Z}$ point increase in energy with increasing strain until they reach the Fermi energy at $\eta=1.87 \%$ [see Fig. 2(b)], while the valence bands at $\mathrm{X}$ remain at the Fermi level. Beyond this value of strain, the valence bands at the $X$ point decrease in energy while they stay at the Fermi level for the $\mathrm{Z}$ point, which constitutes the VBM for the Amm2 phase [Fig. 2(c)].

Concerning the conduction bands, from $\eta=0 \%$, the lowest $d$ bands of the Ti atoms increase in energy at the $\mathrm{Z}$ point and the CBM is then located at the $\Gamma$ point. Figure 3 presents in more details the variations in energy of the three first conduction bands at the $\Gamma$ point. The change in band energy at $\Gamma$ is given as a function of the in-plane strain $\eta$ and of the band character (a more quantitative description of the bands character evolution is done in Fig. 9 in Appendix B). As mentioned previously, the CBM located at $1.64 \mathrm{eV}$ has a $d_{x y}$ character, when PTO is in its equilibrium structure. The two higher conduction bands (labeled CBM +1 and $\mathrm{CBM}+2$ ), with each one a mixed $d_{y z-z x}$ and $d_{y z+z x}$ character, are located 1.07 and $1.13 \mathrm{eV}$ higher in energy [84]. When increasing the in-plane strain, the energy of these two last bands decreases. The energy of the $d_{x y}$ bands, constant in the $P 4 \mathrm{~mm}$ phase, on the contrary starts to increase when PTO adopts the $\mathrm{Cm}$ structure. At a value of strain of $\eta=1.87 \%$, a first crossing happens between the CBM and CBM +1 bands, with no or- bital intermixing. Above this crossing point, the energy of the three sets of bands increases as a function of $\eta$, with a larger slope in the case of the $d_{y z-x z}$ bands. For $\eta=3.6-3.7 \%$, in the $A m m 2$ phase, we can thus observe an anticrossing between the $\mathrm{CBM}+1$ and $\mathrm{CBM}+2$ bands, which is concomitant in this case with a strong hybridization between these two bands.

The variations calculated in the band structure of PTO when we vary $\eta$ are related to the structural changes described in the previous section. The buckling between the oxygen and $\mathrm{Ti}$ atoms associated to the electric polarization tends to increase the energy of bands linked to orbitals lying in planes containing the direction of the electric polarization $\boldsymbol{P}$;

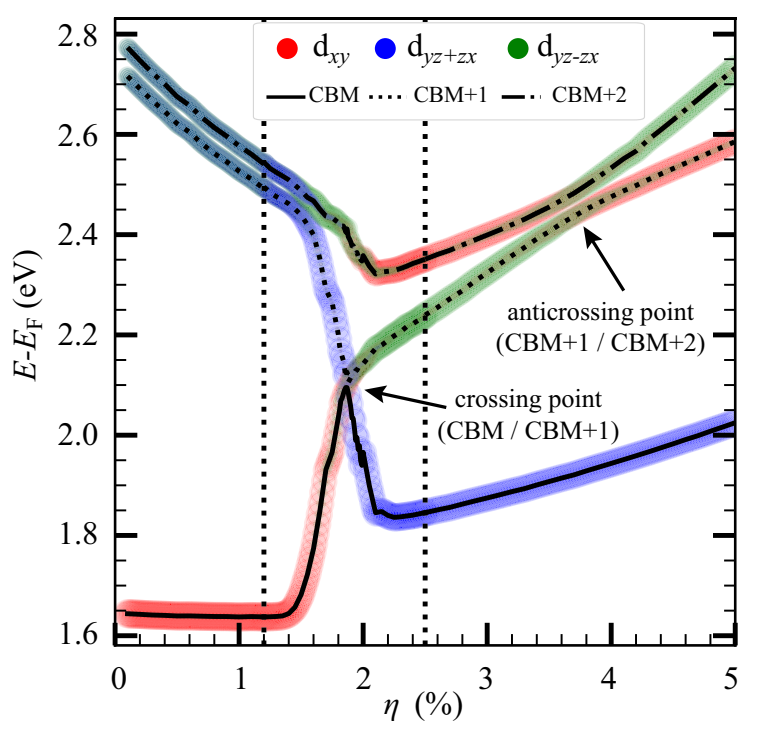

FIG. 3. Evolution of the energy of the three lowest conduction bands located at the $\Gamma$ point as a function of the strain $\eta$. The orbital projections are given with colored circles with the fatness giving the evolution of the band character $n$. The labels "CBM," "CBM +1 ," and "CBM +2 " refer respectively to the first, second, and third conduction bands, ordered by increasing energy. 
orbitals lying in planes perpendicular to $\boldsymbol{P}$ will consequently correspond to bands lower in energy [85]. With the $P 4 \mathrm{~mm}$ phase for which $\boldsymbol{P}$ is out-of-plane, the $d_{x y}$ band represents accordingly the CBM. As seen in Fig. 1(c), the decrease of the polarization with the strain implies a decrease of the buckling, therefore the two sets of degenerated $d_{y z}$ and $d_{z x}$ bands tend to get closer in energy to the $d_{x y}$ band. The tetragonal structure also imposes a similar band character for these CBM +1 and $\mathrm{CBM}+2$ bands, corresponding to a $\Gamma_{5}$ irreducible representation, if we omit the spin-orbit interaction. The rotation of the electric polarization, which starts to become non-negligible from $\eta=1.4 \%$, increases the $d_{x y}$ and decreases the $d_{y z+z x}$ band energies, as can be noticed in Fig. 3. A distinction between the $\mathrm{CBM}+1$ and $\mathrm{CBM}+2$ bands is then established, one having a band character $\left(d_{y z-z x}\right)$ collinear to the in-plane component of $\boldsymbol{P}$, while the other one $\left(d_{y z+z x}\right)$ is perpendicular. At $\eta=1.87 \%$, when the structure of PTO is the closest from the rhombohedral structure $R 3 \mathrm{~m}$, we observe an equivalence between the $\mathrm{Z}$ and the $\mathrm{X}$ points and a degeneracy between the $d_{x y}$ and $d_{y z-z x}$ bands. In the Amm 2 phase, the in-plane electric polarization, oriented along the $[1 \overline{1} 0]$ direction, stabilizes the $d_{y z+z x}$ band as the CBM.

\section{SPIN-ORBIT EFFECTS AND SPIN SPLITTINGS}

In this part we will describe and explain the spin splittings induced in the band structure due to the spin-orbit effect. We will first give a simple description of the evolution as a function of the in-plane strain $\eta$ of these spin splittings and their associated spin textures, as extracted directly from the DFT calculations. We will then analyze these spin-orbit effects more deeply by using a TB approach and by deriving a $\boldsymbol{k} \cdot \boldsymbol{p}$ model.

\section{A. Analysis from the DFT calculations}

\section{Spin splitting in the band structures around the $\Gamma$ point}

As we mentioned in the introduction, the spin-orbitcorrective terms added to the Hamiltonian describing our system strongly depend on the symmetries of this system. To provide an overview of the spin-orbit effects in the different phases of PTO and to make possible a general comparison of the calculated spin splittings between these different phases, we will first describe the variation of spin-dependent band energies difference without considering any symmetrical arguments; the energy difference $\Delta E_{ \pm}(\boldsymbol{k})$ between the two spin states ("+" and "-") can then be fitted by a simple polynomial composed by a linear term in $k$, associated to a coefficient $a_{1}$, and a cubic term with a coefficient $b_{3}$ :

$$
\Delta E_{ \pm}(k)=E_{+}-E_{-}=a_{1} \cdot k+b_{3} \cdot k^{3} .
$$

A more detailed variation of the spin-splitting parameters, which takes into account the symmetries of the space group Amm 2 will be given in Sec. V C. As we found that the linearparameter $\left(a_{1}\right)$ variation displays qualitatively similar trends than the cubic one $\left(b_{3}\right)$, with the appearance of peaks at the same values of $\eta$, only the variation of $a_{1}(\eta)$ calculated near the $\Gamma$ point and along the high-symmetry lines $\Gamma_{\rightarrow \mathrm{M}}$ and $\Gamma_{\rightarrow \mathrm{z}}$ is presented in Figs. 4(a) and 4(b). The variation of $b_{3}(\eta)$ is shown in Appendix C.

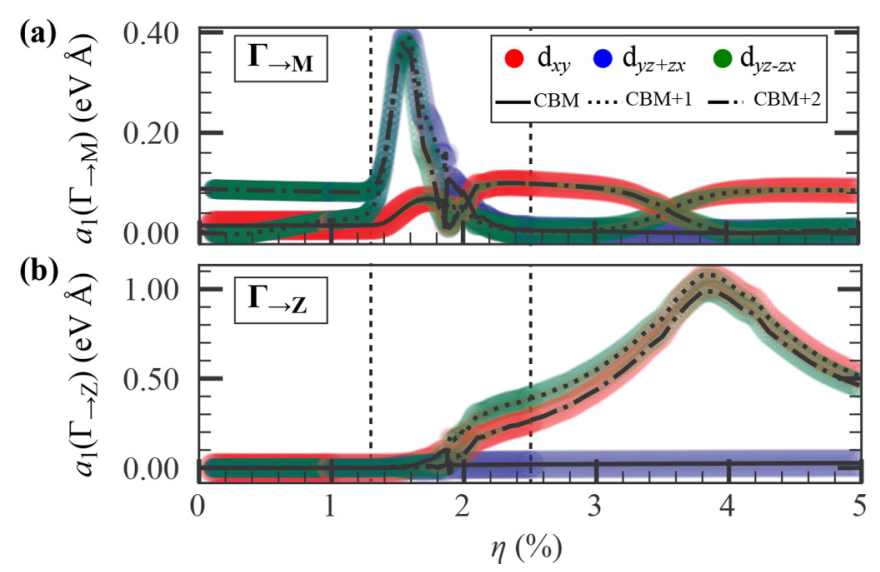

FIG. 4. Variation as a function of the strain $(\eta)$ of the linear coefficient of the spin splitting $a_{1}$ calculated along (a) the $\Gamma$-M-like directions and (b) the $\Gamma$-Z-like directions. The variation of $a_{1}$ along the $\Gamma$-M direction follows the same trend as along the $\Gamma-\mathrm{X}$ direction.

As shown in the tight-binding model proposed in Refs. [16,55] for the P4mm space group, no interaction between $d_{y z}$ and $d_{z x}$ bands is possible, which is in agreement with our calculated low spin splittings for the $d_{y z-z x}$ and $d_{y z+z x}$ bands for $\eta<1.3 \%$, both along the $\Gamma_{\rightarrow \mathrm{M}}$ and $\Gamma_{\rightarrow \mathrm{Z}}$ directions [see Figs. 4(a) and 4(b)].

As displayed previously in Fig. 3, above 1.4\%, when PTO is in the monoclinic $\mathrm{Cm}$ phase, the net distinction between the bands CBM +1 and CBM +2 into $d_{y z-z x}$ and $d_{y z+z x}$ bands allows interactions between them and leads to the increase of the spin-splitting value when the energy difference between these two bands also decreases. As can be seen by comparing Figs. 3 and 4(a), a first peak of the spin splitting characterized by $a_{1}\left(\Gamma_{\rightarrow \mathrm{M}}\right)=0.39 \mathrm{eV} \AA$ is obtained at the $\Gamma$ point when the two bands (CBM +1 and CBM +2$)$ undergo a lift of degeneracy, at $\eta=1.55 \%$ in the direction toward the highsymmetry point $\mathrm{M}$ for the $\mathrm{Cm}$ phase. For the CBM band with a mainly $d_{x y}$ character, a weak spin splitting, ranging from 0.02 to $0.10 \mathrm{eV} \AA$, can also be noticed for the same direction of $\boldsymbol{k}$ and in the same range of $\eta$, because of the reduction of the energy difference between the two interacting $d_{x y}$ and $d_{y z-z x}$ bands above $\eta=1.4 \%$.

From Fig. 4(b), we can see that when increasing the inplane strain further, in the Amm 2 phase, the stronger interplay between the $d_{x y}$ and the $d_{y z-z x}$ [see Fig. 3 and Figs. 9(a) and 9(b) in Appendix B] induces the appearance of a wide peak in the coefficient $a_{1}\left(\Gamma_{\rightarrow \mathrm{Z}}\right)$ around the value of $\eta$ for which the band anticrossing is observed; this peak reaches a maximum value of $a_{1}\left(\Gamma_{\rightarrow \mathrm{z}}\right)=1.08 \mathrm{eV} \AA$ near $\eta=3.8 \%$. The observation of a maximum of the spin-orbit parameter at the avoided crossing between $d$ bands of Ti atoms is consistent with the reported results on $\mathrm{SrTiO}_{3}$-based systems $[55,86]$. It is also interesting to note that the values of the $a_{1}$ coefficient for these $d_{x y}$ and the $d_{y z-z x}$ bands are anisotropic. Indeed, in the Amm2 phase, we can see that they are lower in the $\Gamma_{\rightarrow \mathrm{M}}$ than in the $\Gamma_{\rightarrow \mathrm{z}}$ direction; in the $\Gamma_{\rightarrow \mathrm{M}}$ direction, the variation of $a_{1}\left(\Gamma_{\rightarrow \mathrm{M}}\right)$ is strongly correlated with their character (as can be seen by comparing Figs. 3 and 9 of Appendix B with Fig. 4). 
In this paper, we chose to focus our study on the calculated lowest conduction bands at the $\Gamma$ point, which have a Ti-d band character. Other bands, with a $p$ character, also display a non-negligible spin splitting, with a linear variation in $k$; it is for example the case of the unoccupied $p$ bands of the $\mathrm{Pb}$ atoms. The value of this spin splitting is around $0.2-0.4 \mathrm{eV} \AA$, but in the $\mathrm{Z}_{\rightarrow \mathrm{R}}$ direction, it increases from $a_{1}\left(\mathrm{Z}_{\rightarrow \mathrm{R}}\right)=0.6$ to $0.8 \mathrm{eV} \AA$, for $\eta$ varying from 0 to $1.4 \%$. It then tends to $0 \mathrm{eV} \AA$ when the electric polarization rotates. The linear increase of the spin splitting in the $\mathrm{Z}_{\rightarrow \mathrm{R}}$ direction is continuous with the variation for PTO under compressive strain calculated in our previous study [35] and taking into account that for this phase, we have the relation between the spin-splitting parameter and the linear Rashba coefficient $a_{1}\left(Z_{\rightarrow R}\right)=2 \alpha_{R}\left(Z_{\rightarrow R}\right)$. Concerning the O- $p$ bands constituting the VBM, we have an anisotropic linear spin splitting which is in general approximately of $\sim 0.3 \mathrm{eV} \AA$ along the $\mathrm{X}_{\rightarrow \mathrm{M}}$ or $\mathrm{Z}_{\rightarrow \mathrm{R}}$ directions and $\sim 1 \mathrm{eV} \AA$ along the $\mathrm{X}_{\rightarrow \Gamma}$ or $\mathrm{Z}_{\rightarrow \Gamma}$ directions; in the strain range for which the electric polarization rotates, a negative peak is however observed for the spin-orbit parameters, with a minimum value, respectively, of $0.13 \mathrm{eV} \AA$ and $0.68 \mathrm{eV} \AA$ when $\eta=1.87 \%$.

\section{Spin textures around the $\Gamma$ point}

Figure 5 presents the spin texture of the $\mathrm{CBM}+1$ band as a function of the energy in the $(\Gamma-\mathrm{Z}-\mathrm{A}-\mathrm{M})$ plane near the $\Gamma$ point. This plane orientation is perpendicular to the [1 $\overline{1} 0]$ direction of the electric polarization of the Amm 2 phase. We will now describe how these spin textures vary for different strain values ranging from $\eta=2.5 \%$ to $5 \%$.

For $2.5 \leqslant \eta \leqslant 3.5 \%$, the spins are mostly oriented along the [110] direction [Figs. 5(a)-5(c)], which leads to a quasipersistent spin texture near $\Gamma$. For larger strain, the spins turn when they are the closest to the $\Gamma$ point, i.e., for the highest energies [around 2.5 to $2.6 \mathrm{eV}$ in Figs. 5(d)-5(f)], while they remain oriented along the [110] direction for the lower energies.

From the above discussion, it appears clearly that the $\mathrm{CBM}+1$ band for the Amm 2 phase presents interesting properties such as an enhancement of its spin splitting at the $\Gamma$ point for $\eta=3.8 \%$ and an associated quasipersistent spin texture for lower strain. Using a tight-binding approach and a $\boldsymbol{k} \cdot \boldsymbol{p}$ model, we will now try to get a deeper insight into these properties.

\section{B. Tight-binding approach}

The DFT calculations allowed us to present the general evolution of the spin-orbit effects on the electronic structure. As expected, different variations as a function of the strain are observed, depending on the crystallographic phases. These evolutions could be partly explained by considering a secondorder perturbation theory as proposed in the work of Bahramy et al. [87]. In the framework of a $\boldsymbol{k} \cdot \boldsymbol{p}$ perturbative theory, the authors indeed indicate that the magnitude of the spin splitting is dependent on three parameters: (1) The atomic spin-orbit strength, (2) the energy difference between neighboring electronic states, and (3) the symmetry compatibility between these neighboring states. We then used a TB model to confirm which interactions play a significant role in the

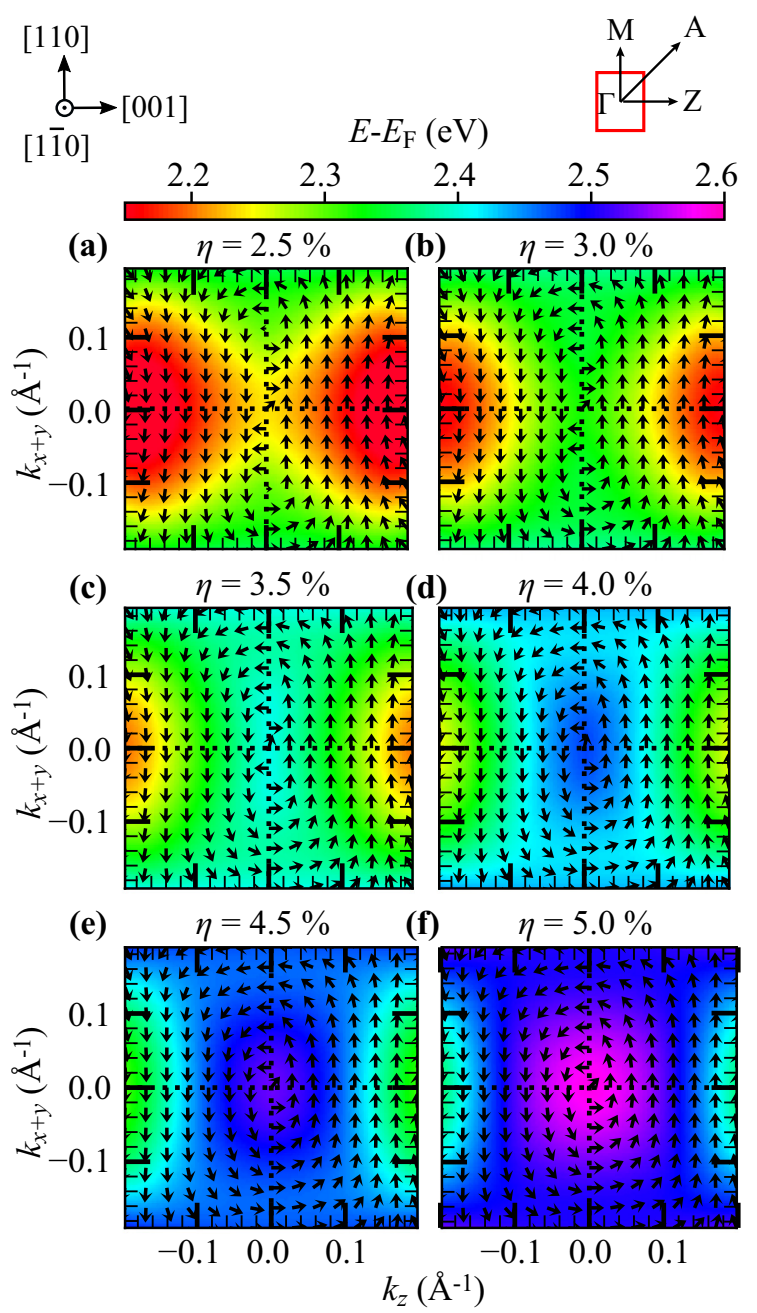

FIG. 5. Spin textures of the CBM +1 band as a function of the energy in the ( $\Gamma$-Z-A-M) plane near the $\Gamma$ point, perpendicular to the electric polarization ([1ㅣㅣ) of the Amm2 phase for different strain values: $\eta=$ (a) $2.5 \%$, (b) $3 \%$, (c) $3.5 \%$, (d) $4 \%$, (e) $4.5 \%$, and (f) $5 \%$. The spins do not have any [1] 0$]$ component.

considered spin-orbit effects. The TB model was built from Wannier functions of the unoccupied $d$ bands of the Ti atoms and $p$ bands of the $\mathrm{Pb}$ atoms.

As it can be seen by comparing Fig. 4(b) and the black curve of Fig. 6, by considering the interactions between these bands, it is possible to recover the same spin splitting value $a_{1}\left(\Gamma_{\rightarrow Z}\right)$ as calculated from the DFT. An advantage of the TB approach is that it is possible to switch off easily the interaction coming from a specific band, as proposed in Ref. [16]. With this method, we hence found that, in addition to the interaction between the CBM +1 and $\mathrm{CBM}+2$ bands, which increases near the anticrossing point, the interactions between the $\mathrm{CBM}+1$ and the $\mathrm{Pb}-p$ bands (and more particularly the $\mathrm{Pb}-p_{x-y}$ bands) are also important. Indeed, if we remove these interactions (red curve), we observe a decrease of the spinsplitting maximum by $31 \%$ and a shift of the peak position from $\eta=3.8 \%$ to $3.6 \%$ corresponding to the band anticrossing; in other words, it also explains why the maximum of the spin splitting $(\eta=3.8 \%)$ is not exactly located at the same value of $\eta$ than the $d$ band anticrossing $(\eta \simeq 3.6 \%)$. The 


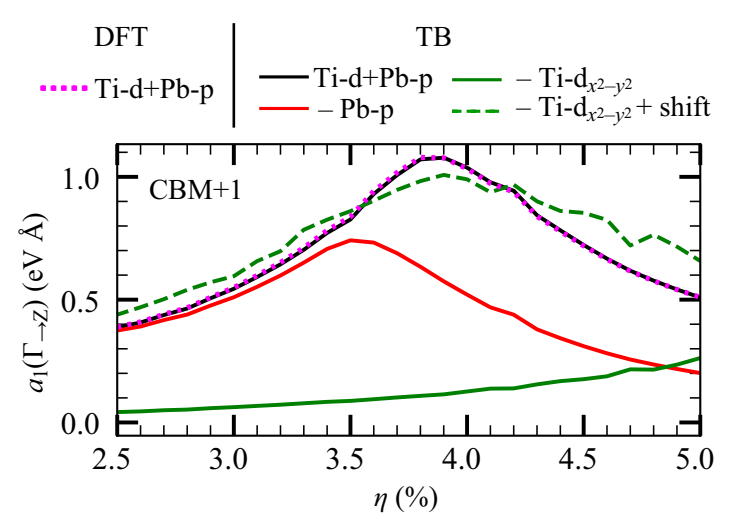

FIG. 6. Evolution of the spin-splitting parameter $a_{1}\left(\Gamma_{\rightarrow Z}\right)$ calculated with the TB model for the band " $\mathrm{CBM}+1$." The black curve has been obtained by taking into account the interaction between all unoccupied Ti- $d$ and $\mathrm{Pb}-p$ bands; this curve matches well with the DFT calculations (magenta dashed line). The solid red and green curves have been calculated, respectively, by removing all the interactions involving the $\mathrm{Pb}-p$ or Ti- $d_{x^{2}-y^{2}}$ bands. The dashed green curve corresponds to a calculation without the Ti- $d_{x^{2}-y^{2}}$ band interactions and in which the $d_{x y}$ band energy has been shifted by applying a potential of $-0.17 \mathrm{eV}$.

interaction between the $d_{x y}$ and $d_{x^{2}-y^{2}}$ bands is also important because it stabilizes the $d_{x y}$ bands by decreasing its energy: If we cancel this interaction, the $d_{x y}$ band is located higher in energy and consequently the anticrossing point is set at the $\Gamma$ point for a higher value of the strain $(>5 \%)$. If we artificially shift this $d_{x y}$ band by applying a potential of $-0.17 \mathrm{eV}$, we find again the same maximum value of the $a_{1}\left(\Gamma_{\rightarrow Z}\right)$ parameter as the case when all interactions are taken into account.

We also used the TB model to confirm the interactions responsible for the peak of the $a_{1}\left(\Gamma_{\rightarrow M}\right)$ parameter in the $C m$ phase (at $\eta=1.55 \%): a_{1}\left(\Gamma_{\rightarrow M}\right)$ first increases because of the increase of the hopping term between the $d_{y z-z x}$ and $d_{y z+z x}$ and then decreases due to an increase of the energy difference between these two bands.

\section{C. $\boldsymbol{k} \cdot \boldsymbol{p}$ model}

Using the theory of invariants (see Appendix D), we derived a $\boldsymbol{k} \cdot \boldsymbol{p}$ model in order to explain the variations of the spin textures calculated as a function of the strain. In particular, we will show why the Amm 2 space group allows the appearance of a persistent spin texture at the $\Gamma$ point. As previously found [35], we confirm in this study that it is mandatory to use a development of this model up to the third order in $\boldsymbol{k}$ near the $\Gamma$ point to reproduce the spin-splitting behavior of the unoccupied $d$ bands of the Ti atoms. We chose to focus our study on the Amm2 structure because this phase presents the stronger spin-splitting values and the most interesting spin textures.

For the $A m m 2$ space group, the high-symmetry point $\Gamma$ is associated to the little group of symmetry $C_{2 v}$, which leads to the following two-bands Hamiltonian:

$$
H_{C_{2 v}}(\boldsymbol{k})=H_{0}(\boldsymbol{k})+H_{x+y}(\boldsymbol{k})+H_{z}(\boldsymbol{k})+H_{x-y}(\boldsymbol{k})
$$

with $H_{0}(\boldsymbol{k})$ being the Hamiltonian part describing the band dispersions and depending on the parameters $\omega_{i}(i=1,2$, or
3) inversely proportional to the effective masses:

$$
H_{0}(\boldsymbol{k})=E_{0}+\omega_{1}\left(k_{x}+k_{y}\right)^{2}+\omega_{2}\left(k_{x}-k_{y}\right)^{2}+\omega_{3} k_{z}^{2}
$$

and the terms $H_{x+y}(\boldsymbol{k}), H_{z}(\boldsymbol{k})$, and $H_{x-y}(\boldsymbol{k})$, which describe the SOC perturbation:

$$
\begin{aligned}
H_{x+y}(\boldsymbol{k})= & \left(\alpha_{1}+\beta_{1} k_{z}^{2}+\gamma_{1}\left(k_{x}+k_{y}\right)^{2}\right. \\
& \left.+\delta_{1}\left(k_{x}-k_{y}\right)^{2}\right) k_{z}\left(\sigma_{x}+\sigma_{y}\right) \\
H_{z}(\boldsymbol{k})= & \left(\alpha_{2}+\beta_{2}\left(k_{x}+k_{y}\right)^{2}+\gamma_{2} k_{z}^{2}\right. \\
& \left.+\delta_{2}\left(k_{x}-k_{y}\right)^{2}\right)\left(k_{x}+k_{y}\right) \sigma_{z} \\
H_{x-y}(\boldsymbol{k})= & \xi\left(k_{x}-k_{y}\right)\left(k_{x}+k_{y}\right) k_{z}\left(\sigma_{x}-\sigma_{y}\right)
\end{aligned}
$$

in which $\alpha_{1 / 2}$ are the spin-orbit parameters linked to the terms linear in $\boldsymbol{k}$ and the $\beta_{1 / 2}, \gamma_{1 / 2}$, and $\delta_{1 / 2}$ parameters to cubic terms. Solving the $H_{x+y}$ Hamiltonian leads to a spin splitting mainly in the $\Gamma_{\rightarrow \mathrm{Z}}$ direction (some anisotropy is given by the terms proportional to $\gamma_{1}$ and $\delta_{1}$ ) and with spins along the [110] directions, for which the sign depends on the sign of $k_{z}$. Similarly, the $H_{z}$ Hamiltonian [Eq. (5b)] is associated to a spin splitting mainly in the $\Gamma_{\rightarrow \mathrm{M}}$ direction and with spins along the [001] directions. The last Hamiltonian [Eq. (5c)] adds a contribution which tends to align the spins along the [11̄0] direction, parallel to the electric polarization.

By solving the Hamiltonian of Eq. (3) for $\boldsymbol{k}$ vectors along the high-symmetry lines $\Gamma_{\rightarrow \mathrm{X}}, \Gamma_{\rightarrow \mathrm{M}}, \Gamma_{\rightarrow \mathrm{z}}$, and $\Gamma_{\rightarrow \mathrm{A} 2}$ [with $A 2=(0.5,-0.5,0.5)]$, it is possible to express the energy difference $\Delta E_{ \pm}$as a function of the parameters $\alpha_{1 / 2}, \beta_{1 / 2}$, and $\delta_{1 / 2}$ :

$$
\begin{aligned}
\Delta E_{\Gamma_{\rightarrow \mathrm{X}}}(k) & =2 \alpha_{2} k+2\left(\beta_{2}+\delta_{2}\right) k^{3} \\
\Delta E_{\Gamma_{\rightarrow \mathrm{M}}}(k) & =2 \sqrt{2} \alpha_{2} k+4 \sqrt{2} \beta_{2} k^{3} \\
\Delta E_{\Gamma_{\rightarrow \mathrm{Z}}}(k) & =2 \sqrt{2} \alpha_{1} k+4 \sqrt{2} \beta_{1} k^{3} \\
\Delta E_{\Gamma_{\rightarrow \mathrm{A}}}(k) & =\frac{2 \sqrt{6}}{3} \alpha_{1} k+\frac{2 \sqrt{6}}{9}\left(\beta_{1}+4 \delta_{1}\right) k^{3} .
\end{aligned}
$$

By making an analogy between Eqs. (6b) and (6c) and the parameters $a_{1}$ and $b_{3}$ from Eq. (2), calculated along the directions $\Gamma_{\rightarrow \mathrm{Z}}$ and $\Gamma_{\rightarrow \mathrm{M}}$, we obtain directly the coefficients $\alpha_{1 / 2}$ and $\beta_{1 / 2}$. The coefficients $\delta_{1 / 2}$ are then trivially deduced from the fits of the band structure using Eqs. (6a) and (6d). The remaining parameters $\gamma_{1 / 2}$ and $\xi$ are calculated by minimizing the sum of the least squares residuals in Eq. (1). The evolution of these parameters as a function of $\eta$ is given for the band CBM +1 in Fig. 7. A comparison between the spin textures calculated using this model and the DFT is given in Appendix E to confirm the validity of the model and of our fitted parameters.

The variations of the linear SOC parameters $\alpha_{1}$ and $\alpha_{2}$ as a function of the strain are presented in Fig. 7(a). Because we have $\alpha_{1}=a_{1}\left(\Gamma_{\rightarrow \mathrm{Z}}\right) / \sqrt{2}, \alpha_{1}$ and $a_{1}\left(\Gamma_{\rightarrow \mathrm{Z}}\right)$ follow the same variation and display a similar peak for $\eta \simeq 3.8 \%$. Similarly, we also have the identity $\alpha_{2}=a_{1}\left(\Gamma_{\rightarrow \mathrm{M}}\right) / \sqrt{2}$; if the value of the $\alpha_{2}$ parameter remains low, it however undergoes an increase by a factor 30 from $\eta=2.5 \%$ to $\eta=5 \%$. The $\alpha_{1}$ term is superior to $\alpha_{2}$ by a factor varying from 100 for $\eta=2.5 \%$, when $\alpha_{2}$ is almost $0 \mathrm{eV} \AA$, to 6 for $\eta=5 \%$, when $\alpha_{2} \simeq 3 \times 10^{-2} \mathrm{eV} \AA$. 


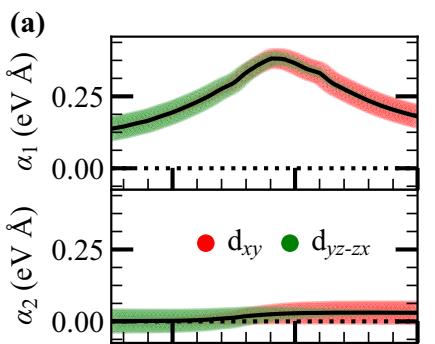

(c)
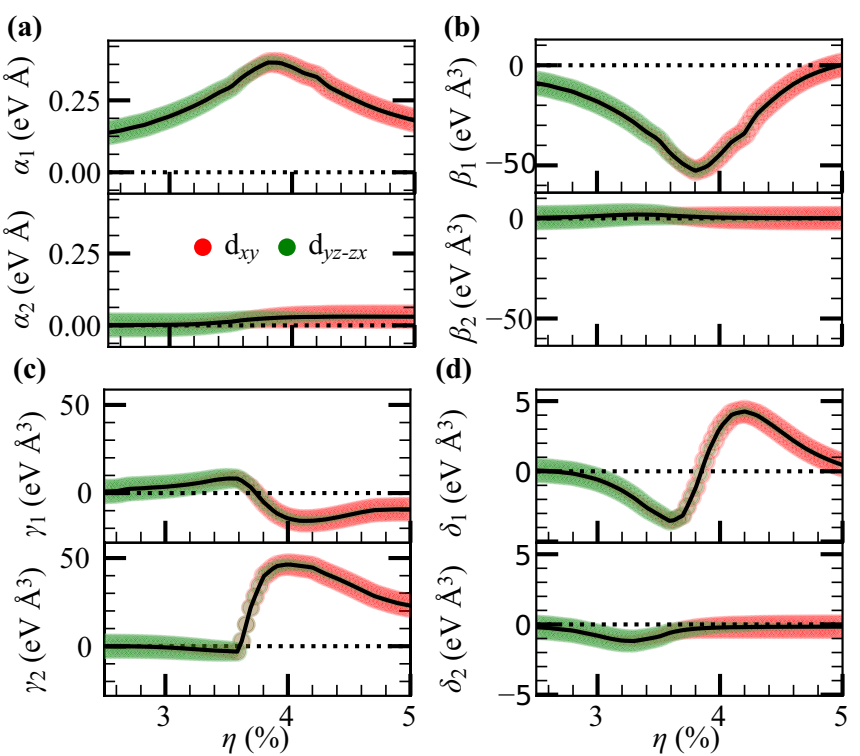

(d)

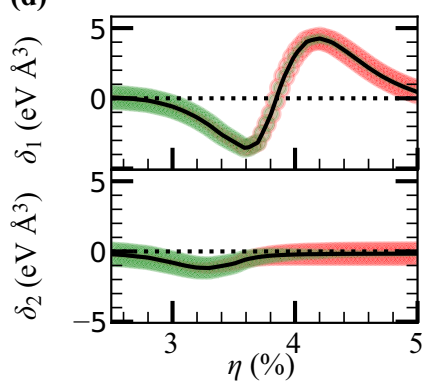

FIG. 7. Variation of the parameters (a) $\alpha_{1 / 2}$, (b) $\beta_{1 / 2}$, (c) $\gamma_{1 / 2}$, and (d) $\delta_{1 / 2}$ as a function of the strain $\eta$ for the CBM +1 band and with orbital projection on $d_{x y}$ (red) and $d_{y z-z x}$ (green). The $\xi$ term has been found to be 0 for each strain.

Regarding the cubic terms, the $\beta_{1}$ parameter [Fig. 7(b)] presents a similar variation to $\alpha_{1}$, with a peak of $-52.7 \mathrm{eV} \AA^{3}$ at $\eta=3.8 \%$. As in the case of $\alpha_{1 / 2}, \beta_{1}$ is superior to $\beta_{2}$, in particular around the band anticrossing, at $\eta=3.8 \%$, where the $\beta_{1} / \beta_{2}$ ratio is nearly equal to $100 . \gamma_{1}$ and $\gamma_{2}$ [Fig. 7(c)] display weak variations until the band crossing at $\eta=3.6 \%$, where we observe a strong variation of each term for higher strain; these values increase up to $46.4 \mathrm{eV} \AA^{3}$ at $\eta=4 \%$ for $\gamma_{2}$ and up to $-15.8 \mathrm{eV} \AA^{3}$ at $\eta=4.1 \%$ for $\gamma_{1}$. Finally, the $\xi$ term has been found to be nearly 0 in our calculation, implying that we can neglect the $H_{x-y}$ Hamiltonian (which is even strictly 0 along the high-symmetry lines of Eqs. 6(a) and 6(d)].

To summarize, for an in-plane strain $\eta$ inferior to $3.6 \%$, the set of coefficients $\left(\alpha_{1}, \beta_{1}, \gamma_{1}, \delta_{1}\right)$ associated with the Hamiltonian $H_{x+y}(\boldsymbol{k})$ for the band CBM +1 gives a dominant contribution to the spin splitting in comparison with the set of coefficients $\left(\alpha_{2}, \beta_{2}, \gamma_{2}, \delta_{2}\right)$ of the Hamiltonian $H_{z}(\boldsymbol{k})$. This statement remains true below the bands anticrossing, while the band CBM +1 possesses a mainly $d_{y z-z x}$ character, and it leads to the observed alignment of the spins along a [110] direction, near the $\Gamma$ point. Above the band anticrossing at $\eta=3.6-3.7 \%$, the quasipersistent spin texture behavior is transferred to the band CBM +2 (as can be seen in Fig. 13 of Appendix F), while the band CBM +1 becomes mainly $d_{x y}$. The Hamiltonian $H_{z}$, which tends to align the spins along the [001] direction, is then non-negligible for the CBM +1 band because of (i) the increase of the $\alpha_{2}$ parameter, which becomes non-negligible in comparison to $\alpha_{1}$, and (ii) the strong increase of $\gamma_{2}$. We observe in consequences a rotation of the spins near the $\Gamma$ point.

The above-mentioned variations of each coefficient is consistent with previous reports in the literature $[18,26]$, which state that a persistent spin texture can be achieved when the linear Rashba and Dresselhaus parameters are equal. It is indeed always possible to be as near as possible from the
$\Gamma$ point, so that the cubic terms in $\boldsymbol{k}$ will be negligible. By changing our basis such as $x \equiv\left[\frac{\sqrt{2}}{2}, \frac{\sqrt{2}}{2}, 1\right], y \equiv\left[\frac{\sqrt{2}}{2}, \frac{\sqrt{2}}{2}, \overline{1}\right]$, and $z \equiv[1 \overline{1} 0]$, it is possible to show that we can express our coefficients $\alpha_{1}$ and $\alpha_{2}$ as a function of linear Dresselhaus and Rashba-like terms: $\alpha_{\mathrm{D}}=-\frac{1}{\sqrt{2}}\left(\alpha_{1}+\alpha_{2}\right)$ and $\alpha_{\mathrm{R}}=$ $\frac{1}{\sqrt{2}}\left(\alpha_{2}-\alpha_{1}\right)$. For $2.5 \leqslant \eta \leqslant 3.6 \%$, when $\alpha_{2} \simeq 0 \mathrm{eV} \AA$, we then indeed have $\alpha_{R} \sim \alpha_{D}$, which is in agreement with the obtained persistent spin texture. At $\eta=3.8 \%$, when the spin splitting is maximum, the values of the linear Rashba and Dresselhaus parameters are, respectively, $\alpha_{\mathrm{D}}=-0.29 \mathrm{eV} \AA$ and $\alpha_{\mathrm{R}}=-0.25 \mathrm{eV} \AA$.

\section{CONCLUSION}

In summary, by using first-principles calculations, we studied the effect of an applied in-plane tensile strain $\eta$ on the structural and electronic properties of the well-known ferroelectric oxide $\mathrm{PbTiO}_{3}$. Our results confirm that this tensile strain induces a rotation of the electric polarization from an out-of-plane to an in-plane direction, changing then the space group of the material, which varies from a tetragonal, to a monoclinic, and finally an orthorhombic structure.

We predicted that the calculated structural changes result in variations of the electronic structure and the spin textures originating from the spin-orbit interaction. We focused our study on the conduction bands, the lowest in energy at the highsymmetry point $\Gamma$. We have shown that the spin splittings of the bands can be increased in the $C m$ phase, for a value of $\eta$ for which the electric polarization starts to rotate and the energy differences between the bands CBM +1 and $\mathrm{CBM}+2$ is still low. A second peak in the spin-splitting energy difference is observed for $\eta=3.8 \%$, when the avoided crossing between the strongly hybridized $d_{x y}$ and $d_{y z-z x}$ bands is located near the $\Gamma$ point. For this value of $\eta$, the largest spin-orbit coefficients near $\Gamma$ and in the $\Gamma-\mathrm{Z}$ direction are obtained, i.e., $a_{1}=1.08$ $\mathrm{eV} \AA$ and $b_{3}=-75.2 \mathrm{eV} \AA^{3}$. We demonstrated that the maximum of the linear term $a_{1}$ corresponds to a combination of a linear Rashba and a linear Dresselhaus parameters of $\alpha_{\mathrm{D}}=$ $-0.29 \mathrm{eV} \AA$ and $\alpha_{\mathrm{R}}=-0.25 \mathrm{eV} \AA$. This phenomenon is reminiscent of the spin-orbit effects increase, both predicted and measured, at the band anticrossing of Ti- $d$ bands in $\mathrm{SrTiO}_{3}$ based systems $[55,86]$. In addition to the large spin splitting linked to $A m m 2$ structure, the $C_{2 v}$ little group of $\Gamma$ allows the stabilization of a quasipersistent spin texture associated with the $d_{y z-z x}$ band. The combination of these two properties is highly desirable to preserve spin-dependent information and guarantee large spin-to-charge current conversions.

An interesting perspective for this work would be to extend the study to other $\boldsymbol{k}$ points than $\Gamma$, for which the band anticrossing may be also present for other values of strain and also to calculate what would be the influence of the spin-orbit effects on transport properties. We also expect that our results obtained on a pristine and simple material could be extended to other more complex $\operatorname{Ti}\left(d^{0}\right)$-based systems.

\section{ACKNOWLEDGMENTS}

This study has been partially supported through the EUR Grant NanoX No. ANR-17-EURE-0009 in the framework of the Programme des Investissements d'Avenir. H.J., C.P., and 

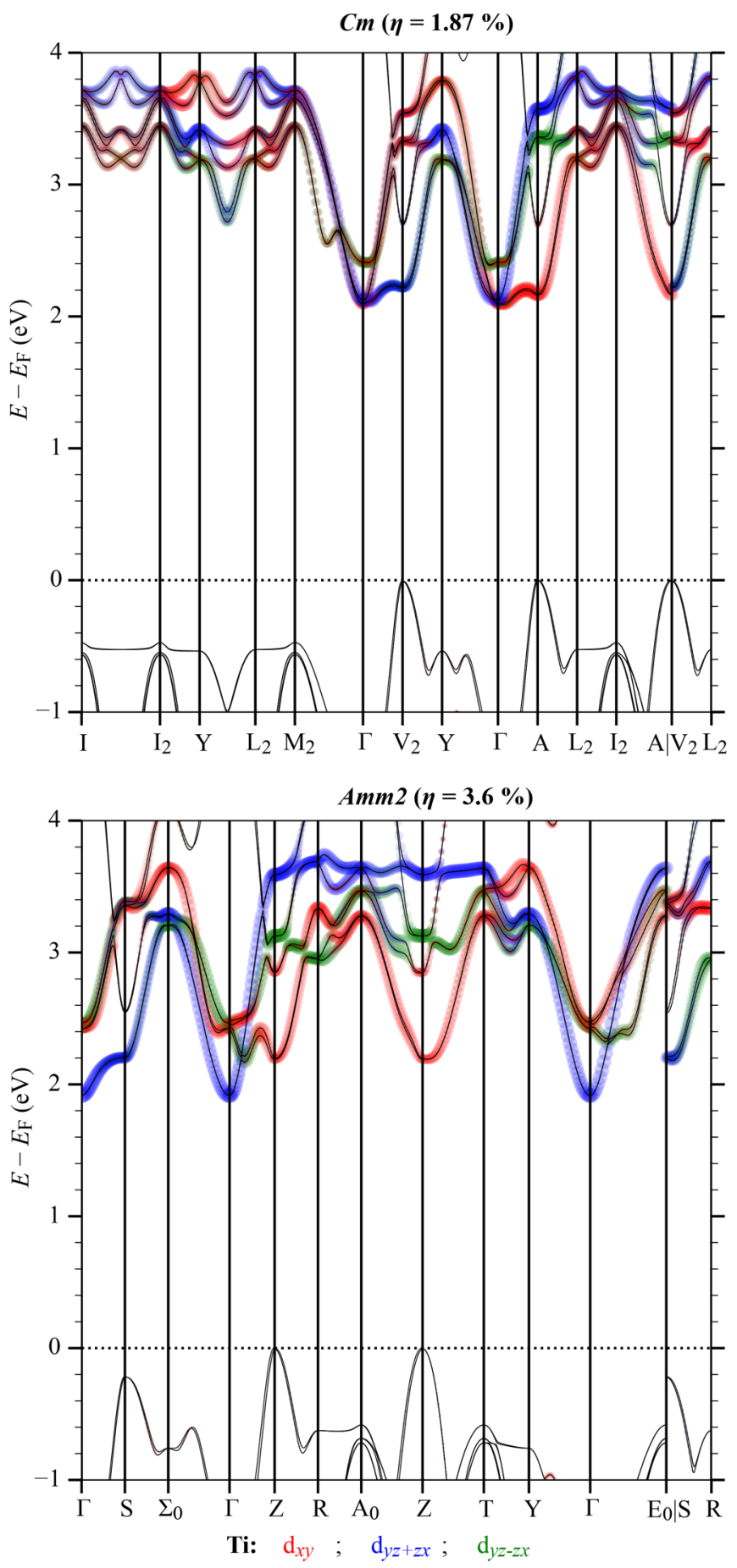

FIG. 8. Band structure calculated for the $C m$ phase at $\eta=1.87 \%$ (top) and the Amm 2 phase at $\eta=3.6 \%$ (bottom). The Ti $(d)$-orbital contributions are shown in color.

L.B. thank the Vannevar Bush Faculty Fellowship (VBFF) from the Department of Defense. This work was granted access to the HPC resources of CALMIP (Allocation No. 2019-2020/P1229).

\section{APPENDIX A: DETAILS ABOUT THE BAND STRUCTURES}

As mentioned in the section of the computational details (Sec. II), in order to simplify our discussion about the variation of each calculated parameter, we kept the same notations
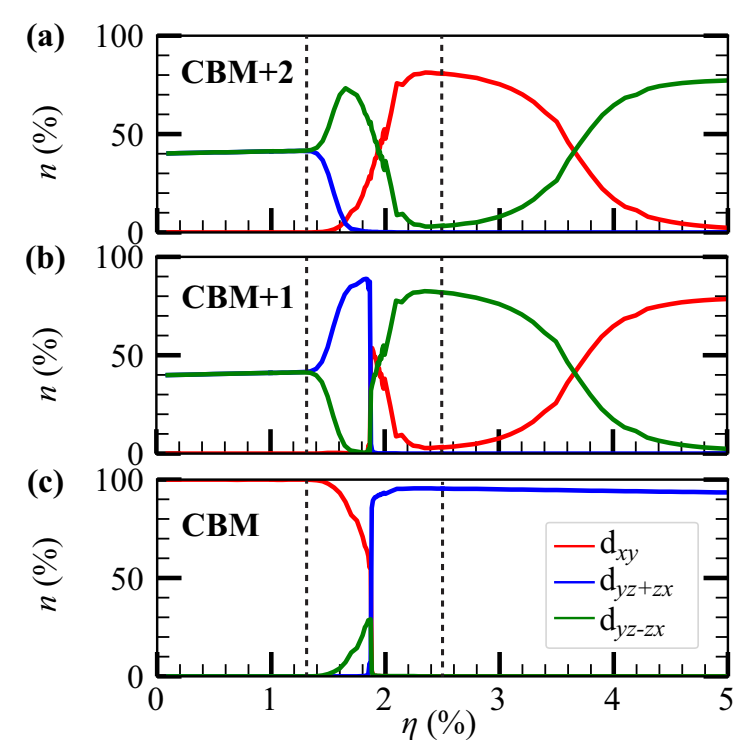

FIG. 9. Evolution of the band character $n$ obtained by projecting the wave functions onto spherical harmonics, at the $\Gamma$ point, and as a function of the strain $\eta$, for the three first conduction bands (a) $\mathrm{CBM}+2$, (b) $\mathrm{CBM}+1$, and (c) CBM.

to label the high-symmetry points. The equivalences between our notations and the standard notations provided by the Bilbao Crystallographic Server [67,68] is given in Table II; these equivalences can be found by suitably choosing the reference axes and omitting the distortions which differentiate the $P 4 m m, C m$, and $A m m 2$ phases.

In addition, Fig. 8 provides the complete band structures for the $\mathrm{Cm}$ and $\mathrm{Amm} 2$ phases. Theses band structures display some high-symmetry points omitted in Fig. 2, either because they are very similar to other high-symmetry points or because the conduction bands are much higher in energy than the CBM.

(a)

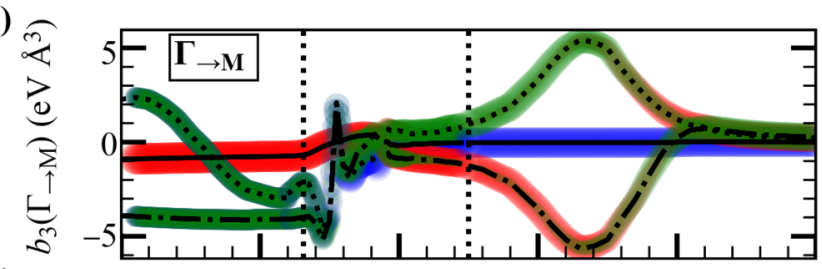

(b)

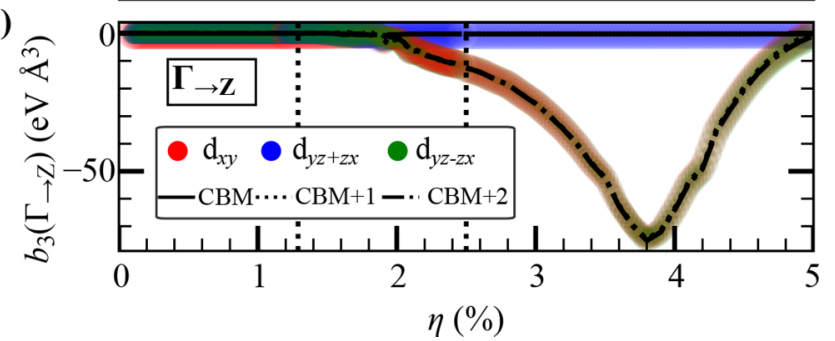

FIG. 10. Cubic coefficients of the spin splitting $b_{3}$ as a function of the in-plane strain $\eta$, with orbital projection and along (a) the $\Gamma_{\rightarrow \mathrm{M}}$ and (b) the $\Gamma_{\rightarrow \mathrm{z}}$ direction. The results calculated for the $\Gamma_{\rightarrow \mathrm{M}}$ direction are the same as those for the $\Gamma_{\rightarrow \mathrm{x}}$ direction. 


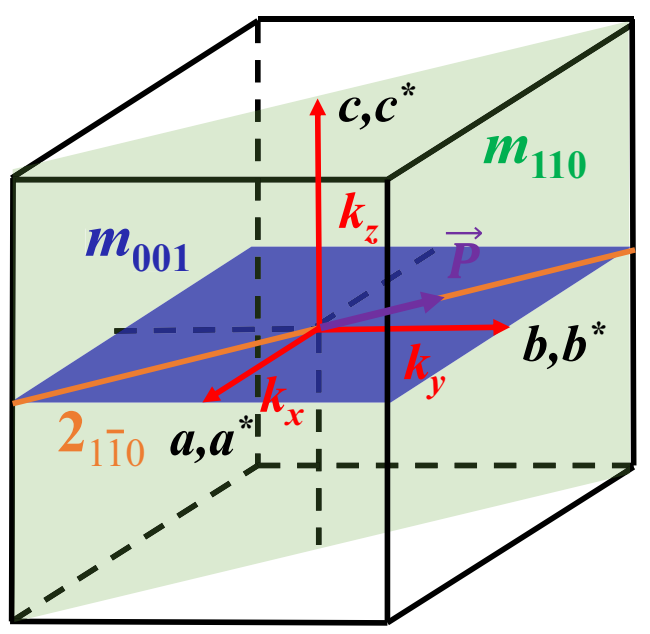

FIG. 11. Sketch of the symmetry rotation axis and mirror planes of the $C_{2 v}$ point group.

\section{APPENDIX B: Ti $(d)$-BANDS HYBRIDIZATION}

Figure 9 shows the variation as a function of $\eta$ of the orbital projections for the three first conduction bands. This figure is intended to help the reader for the interpretation of Figs. 3, 4, and 7, in which the band character is represented by the fatness of the curves.

\section{APPENDIX C: VARIATION OF THE PARAMETER $b_{3}(\eta)$}

Figure 10 presents the evolution of the cubic part of regression of the spin energy difference as a function of the strain along the $\Gamma_{\rightarrow \mathrm{M}}$ and the $\Gamma_{\rightarrow \mathrm{Z}}$ directions. In the $\Gamma_{\rightarrow \mathrm{M}}$ direction, three peaks can be observed, two for the $\mathrm{Cm}$ phase and one for the $A m m 2$ phase: The first peak of $-5.2 \mathrm{eV} \AA^{3}$ appears at $\eta=1.45 \%$, the second one of $2.07 \mathrm{eV} \AA^{3}$ is present at $\eta=1.55 \%$ and finally, the last peak with a magnitude of $5.5 \mathrm{eV} \AA^{3}$ at $\eta=3.30 \%$ displays opposite signs for the $d_{y z-z x}$ and $d_{x y}$ bands. In the $\Gamma_{\rightarrow z}$ direction, as the $a_{1}$ parameter, the variation of $b_{3}(\eta)$ presents only a wide peak with a maximum value of $-75.2 \mathrm{eV}^{3}$ at $\eta=3.8 \%$.

\section{APPENDIX D: SYMMETRIES OF THE Amm2 SPACE GROUP}

The $\boldsymbol{k} \cdot \boldsymbol{p}$ model of Eqs. (3), (4), and (5) has been derived using the theory of invariants. The Amm 2 space group

TABLE III. Symmetry table of the $C_{2 v}$ point group. The twofold rotation axis $\mathbf{2}_{\mathbf{1 1 0}}$ and the mirror planes $\boldsymbol{m}_{\mathbf{1 1 0}}$ and $\boldsymbol{m}_{\mathbf{0 0 1}}$ are shown in Fig. 11.

\begin{tabular}{lcccc}
\hline \hline $\boldsymbol{C}_{\mathbf{2}}$ & $\mathbf{2}_{\mathbf{1} \mathbf{0}}$ & $\boldsymbol{m}_{\mathbf{1 1 0}}$ & $\boldsymbol{m}_{\mathbf{0 0 1}}$ & $\boldsymbol{T}$ \\
\hline $\boldsymbol{k}_{\boldsymbol{x}}$ & $-k_{y}$ & $-k_{y}$ & $k_{x}$ & $-k_{x}$ \\
$\boldsymbol{k}_{\boldsymbol{y}}$ & $-k_{x}$ & $-k_{x}$ & $k_{y}$ & $-k_{y}$ \\
$\boldsymbol{k}_{z}$ & $-k_{z}$ & $k_{z}$ & $-k_{z}$ & $-k_{z}$ \\
$\boldsymbol{\sigma}_{\boldsymbol{x}}$ & $-\sigma_{y}$ & $\sigma_{y}$ & $-\sigma_{x}$ & $-\sigma_{x}$ \\
$\boldsymbol{\sigma}_{\boldsymbol{y}}$ & $-\sigma_{x}$ & $\sigma_{x}$ & $-\sigma_{y}$ & $-\sigma_{y}$ \\
$\boldsymbol{\sigma}_{z}$ & $-\sigma_{z}$ & $-\sigma_{z}$ & $\sigma_{z}$ & $-\sigma_{z}$ \\
\hline \hline
\end{tabular}

DFT k.p Model

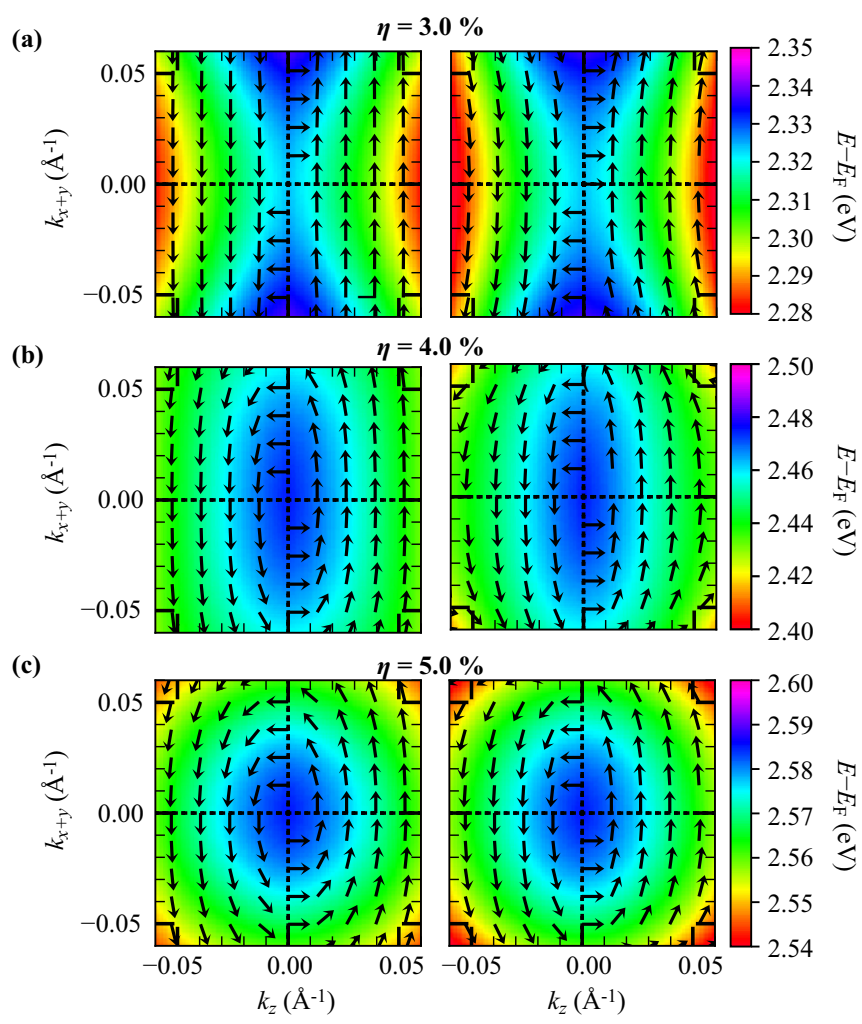

FIG. 12. Spin textures for the CBM +1 band, as calculated from DFT (left) or from the $\boldsymbol{k} \cdot \boldsymbol{p}$ model (right) described in Sec. V C. The color scale gives the energy of the considered band. The spin textures are plotted in a $(\Gamma-\mathrm{Z}-\mathrm{A}-\mathrm{M})$ plane that is perpendicular to the electric polarization.

describes a orthorhombic structure with the electric polarization oriented along the [110] direction. The little group corresponding to the high-symmetry point $\Gamma$ is $C_{2 v}$, which
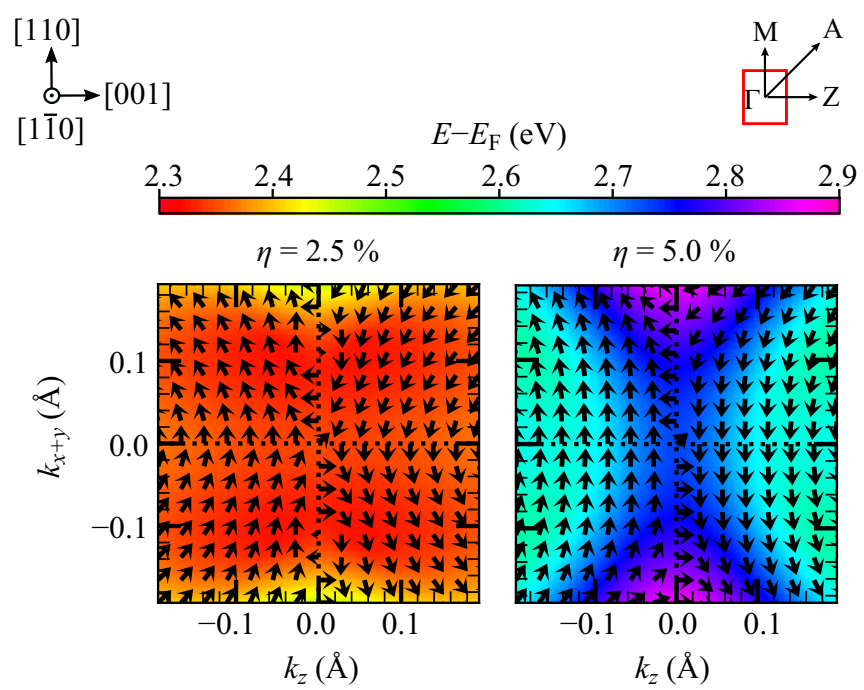

FIG. 13. Spin textures calculated with the DFT for the third conduction band $(\mathrm{CBM}+2)$ for an in-plane strain of $\eta=2.5 \%$ (left) and $\eta=5.0 \%$ (right). 
possesses a twofold axis of rotation denoted as $2_{1 \overline{1} 0}$ and two mirror planes $m_{110}$ and $m_{001}$ (see Fig. 11). The time symmetry is labeled $T$. The symmetry transformations of the vector components of the wave vector $\boldsymbol{k}$ and the Pauli matrices $\sigma$ are given in Table III.

\section{APPENDIX E: COMPARISON BETWEEN THE DFT CALCULATIONS AND THE $k \cdot p$ MODEL}

Figure 12 shows that the $\boldsymbol{k} \cdot \boldsymbol{p}$ model proposed in Sec. V C for the CBM +1 band and the fitted spin-orbit parameters allow us to recover the spin textures obtained directly from the DFT calculations: The model is in agreement with the DFT calculations both for the band energy and the spin orientations.

\section{APPENDIX F: SPIN TEXTURE OF THE THIRD CONDUCTION BAND $(\mathrm{CBM}+2)$}

Figure 13 shows the spin texture corresponding to the conduction band labeled CBM +2 for two strain states of the Amm2 structural phase of PTO. The in-plane strain values are $\eta=2.5 \%$ and $\eta=5.0 \%$, i.e., respectively below and above the value of $\eta=3.8 \%$ for which the band anticrossing is observed at the $\Gamma$ point. When compared with Fig. 5, which displays the spin texture associated with the conduction band $\mathrm{CBM}+1$, we can see that the CBM +2 band has an opposite behavior, with a quasipersistent spin texture appearing above the anticrossing point. At this anticrossing point, the band character of the CBM +2 band switches from mostly $d_{x y}$ to $d_{y z-z x}$, which confirms that the latter is intimately linked with the alignment of the spins parallel to the [110] direction.
[1] S. Datta and B. Das, Appl. Phys. Lett. 56, 665 (1990).

[2] S. Manipatruni, D. E. Nikonov, C.-C. Lin, T. A. Gosavi, H. Liu, B. Prasad, Y.-L. Huang, E. Bonturim, R. Ramesh, and I. A. Young, Nature (London) 565, 35 (2019).

[3] D. Bercioux and P. Lucignano, Rep. Prog. Phys. 78, 106001 (2015).

[4] A. Manchon, H. C. Koo, J. Nitta, S. M. Frolov, and R. A. Duine, Nat. Mater. 14, 871 (2015).

[5] L. L. Tao, T. R. Paudel, A. A. Kovalev, and E. Y. Tsymbal, Phys. Rev. B 95, 245141 (2017).

[6] A. Manchon, J. Železný, I. M. Miron, T. Jungwirth, J. Sinova, A. Thiaville, K. Garello, and P. Gambardella, Rev. Mod. Phys. 91, 035004 (2019).

[7] G. Dresselhaus, Phys. Rev. 100, 580 (1955).

[8] M. I. Dyakonov, Spin physics in semiconductors (SpringerVerlag, Berlin, Heidelberg, 2008), Chap. 1, Basics of Semiconductor and Spin Physics.

[9] Y. A. Bychkov and E. I. Rashba, JETP Lett. 39, 78 (1984).

[10] E. I. Rashba and V. I. Sheka, Fiz. Tverd. Tela 1(2), 162 (1959).

[11] R. Winkler, Spin-Orbit Coupling Effects in Two-Dimensional Electron and Hole Systems (Springer, Berlin, Heidelberg, 2003).

[12] L. Petersen and P. Hedegård, Surf. Sci. 459, 49 (2000).

[13] F. Reinert, G. Nicolay, S. Schmidt, D. Ehm, and S. Hüfner, Phys. Rev. B 63, 115415 (2001).

[14] A. D. Caviglia, M. Gabay, S. Gariglio, N. Reyren, C. Cancellieri, and J.-M. Triscone, Phys. Rev. Lett. 104, 126803 (2010).

[15] L. L. Tao and J. Wang, J. Appl. Phys. 120, 234101 (2016).

[16] H. Djani, A. C. Garcia-Castro, W.-Y. Tong, P. Barone, E. Bousquet, S. Picozzi, and P. Ghosez, npj Quantum Mater. 4, 51 (2019).

[17] S. Vajna, E. Simon, A. Szilva, K. Palotas, B. Ujfalussy, and L. Szunyogh, Phys. Rev. B 85, 075404 (2012).

[18] S. D. Ganichev and L. E. Golub, Phys. Status Solidi (b) 251, 1801 (2014).

[19] M. I. Dyakonov and V. I. Perel, Phys. Lett. A 35, 459 (1971).

[20] J. Sinova, S. O. Valenzuela, J. Wunderlich, C. H. Back, and T. Jungwirth, Rev. Mod. Phys. 87, 1213 (2015).

[21] A. G. Aronov and Y. B. Lyanda-Geller, JETP Lett. 50, 431 (1989).
[22] V. M. Edelstein, Solid State Commun. 73, 233 (1990).

[23] S. D. Ganichev, E. L. Ivchenko, V. V. Bel'kov, S. A. Tarasenko, M. Sollinger, D. Weiss, W. Wegscheider, and W. Prettl, Nature (London) 417, 153 (2002).

[24] S. Bhattacharjee, S. Singh, D. Wang, M. Viret, and L. Bellaiche, J. Phys.: Condens. Matter 26, 315008 (2014).

[25] M. I. D’yakonov and V. I. Perel', Fiz. Tverd. Tela. 13, 3581 (1971) [Soviet Physics Solid State, Ussr 13(12), 3023 (1972)].

[26] L. L. Tao and E. Y. Tsymbal, Nat. Commun. 9, 2763 (2018).

[27] H. J. Zhao, H. Nakamura, R. Arras, C. Paillard, P. Chen, J. Gosteau, X. Li, Y. Yang, and L. Bellaiche, Phys. Rev. Lett. 125, 216405 (2020).

[28] D. D. Sante, P. Barone, R. Bertacco, and S. Picozzi, Adv. Mater. 25, 509 (2013).

[29] M. Liebmann, C. Rinaldi, D. D. Sante, J. Kellner, C. Pauly, R. N. Wang, J. E. Boschker, A. Giussani, S. Bertoli, M. Cantoni, L. Baldrati, M. Asa, I. Vobornik, G. Panaccione, D. Marchenko, J. Sánchez-Barriga, O. Rader, R. Calarco, S. Picozzi, R. Bertacco, and M. Morgenstern, Adv. Mater. 28, 560 (2016).

[30] C. Rinaldi, S. Varotto, M. Asa, J. Sławińska, J. Fujii, G. Vinai, S. Cecchi, D. Di Sante, R. Calarco, I. Vobornik, G. Panaccione, S. Picozzi, and R. Bertacco, Nano Lett. 18, 2751 (2018).

[31] S. Picozzi, Front. Phys. 2, 10 (2014).

[32] L. G. D. da Silveira, P. Barone, and S. Picozzi, Phys. Rev. B 93, 245159 (2016).

[33] D. Di Sante, P. Barone, A. Stroppa, K. F. Garrity, D. Vanderbilt, and S. Picozzi, Phys. Rev. Lett. 117, 076401 (2016).

[34] J. Varignon, J. Santamaria, and M. Bibes, Phys. Rev. Lett. 122, 116401 (2019).

[35] R. Arras, J. Gosteau, H. J. Zhao, C. Paillard, Y. Yang, and L. Bellaiche, Phys. Rev. B 100, 174415 (2019).

[36] H. J. Zhao, P. Chen, C. Paillard, R. Arras, Y.-W. Fang, X. Li, J. Gosteau, Y. Yang, and L. Bellaiche, Phys. Rev. B 102, 041203(R) (2020).

[37] M. A. U. Absor, H. Kotaka, F. Ishii, and M. Saito, Appl. Phys. Express 7, 053002 (2014).

[38] N. Yamaguchi and F. Ishii, Appl. Phys. Express 10, 123003 (2017).

[39] B. Zhou, Nanoscale 12, 5533 (2020). 
[40] M. Okuyama and Y. Hamakawa, Int. J. Eng. Sci. 29, 391 (1991).

[41] N. Setter, D. Damjanovic, L. Eng, G. Fox, S. Gevorgian, S. Hong, A. Kingon, H. Kohlstedt, N. Y. Park, G. B. Stephenson, I. Stolitchnov, A. K. Taganstev, D. V. Taylor, T. Yamada, and S. Streiffer, J. Appl. Phys. 100, 051606 (2006).

[42] S. Shujun Zhang, F. Li, J. Luo, R. Sahul, and T. R. Shrout, IEEE Trans. Ultrason. Ferroelectr. Freq. Control. 60, 1572 (2013)

[43] L. W. Martin and A. M. Rappe, Nat. Rev. Mater. 2, 16087 (2016).

[44] L. Sun, Y. Chen, W. Ma, L. Wang, T. Yu, M. Zhang, and N. Ming, Appl. Phys. Lett. 68, 3728 (1996).

[45] R. Schafranek, S. Li, F. Chen, W. Wu, and A. Klein, Phys. Rev. B 84, 045317 (2011).

[46] X. He and K.-j. Jin, Phys. Rev. B 94, 224107 (2016).

[47] H. J. Zhao, A. Filippetti, C. Escorihuela-Sayalero, P. Delugas, E. Canadell, L. Bellaiche, V. Fiorentini, and J. Íñiguez, Phys. Rev. B 97, 054107 (2018).

[48] C. Paillard, E. Torun, L. Wirtz, J. Íñiguez, and L. Bellaiche, Phys. Rev. Lett. 123, 087601 (2019).

[49] P. Ghosez and K. M. Rabe, Appl. Phys. Lett. 76, 2767 (2000).

[50] B. Meyer and D. Vanderbilt, Phys. Rev. B 63, 205426 (2001).

[51] M. Stengel, D. Vanderbilt, and N. A. Spaldin, Nat. Mater. 8, 392 (2009).

[52] Y. Umeno, J. M. Albina, B. Meyer, and C. Elsässer, Phys. Rev. B 80, 205122 (2009).

[53] B. Yin and S. Qu, J. Appl. Phys. 114, 063703 (2013).

[54] R. Dorin, L. D. Filip, L. Pintilie, K. T. Butler, and N. Plugaru, New J. Phys. 21, 113005 (2019).

[55] Z. Zhong, A. Tóth, and K. Held, Phys. Rev. B 87, 161102(R) (2013).

[56] K. V. Shanavas, Phys. Rev. B 93, 045108 (2016).

[57] Y. Yang, M. Stengel, W. Ren, X. H. Yan, and L. Bellaiche, Phys. Rev. B 86, 144114 (2012).

[58] C. Paillard, S. Prokhorenko, and L. Bellaiche, npj Comput. Mater. 5, 1 (2019).

[59] N. A. Pertsev, A. G. Zembilgotov, and A. K. Tagantsev, Phys. Rev. Lett. 80, 1988 (1998).

[60] L. Chen, Y. Yang, Z. Gui, D. Sando, M. Bibes, X. K. Meng, and L. Bellaiche, Phys. Rev. Lett. 115, 267602 (2015).

[61] G. Kresse and J. Hafner, Phys. Rev. B 49, 14251 (1994).

[62] G. Kresse and J. Furthmüller, Phys. Rev. B 54, 11169 (1996).

[63] P. E. Blöchl, Phys. Rev. B 50, 17953 (1994).

[64] G. I. Csonka, J. P. Perdew, A. Ruzsinszky, P. H. T. Philipsen, S. Lebègue, J. Paier, O. A. Vydrov, and J. G. Ángyán, Phys. Rev. B 79, 155107 (2009).

[65] H. J. Monkhorst and J. D. Pack, Phys. Rev. B 13, 5188 (1976).

[66] S. Steiner, S. Khmelevskyi, M. Marsmann, and G. Kresse, Phys. Rev. B 93, 224425 (2016).

[67] M. I. Aroyo, A. Kirov, C. Capillas, J. M. Perez-Mato, and H. Wondratschek, Acta Crystallogr. Sect. A 62, 115 (2006).

[68] M. I. Aroyo, J. M. Perez-Mato, C. Capillas, E. Kroumova, S. Ivantchev, G. Madariaga, A. Kirov, and H. Wondratschek, Z. Kristallogr. - Cryst. Mater. 221, 15 (2009).
[69] G. F. Koster, J. D. Dimmock, R. G. Wheeler, and H. Statz, Properties of the Thirty-Two Point Groups, edited by G. F. Koster (M.I.T. Press, Cambridge, MA, 1963).

[70] S. L. Altmann and P. Herzig, Point-Group Theory Tables (Clarendon Press, Oxford; Oxford University Press, New York, 1994).

[71] L. C. Lew Yan Voon and M. Willatzen, The $k$ p Method (Springer-Verlag, Berlin, Heidelberg, 2009).

[72] A. A. Mostofi, J. R. Yates, G. Pizzi, Y.-S. Lee, I. Souza, D. Vanderbilt, and N. Marzari, Comput. Phys. Commun. 185, 2309 (2014).

[73] H. T. Stokes and D. M. Hatch, J. Appl. Crystallogr. 38, 237 (2005).

[74] H. T. Stokes, D. M. Hatch, and B. J. Campbell., FINDSYM, ISOTROPY Software Suite, iso.byu.edu (2005).

[75] R. D. King-Smith and D. Vanderbilt, Phys. Rev. B 47, 1651 (1993).

[76] G. Shirane, S. Hoshino, and K. Suzuki, Phys. Rev. 80, 1105 (1950).

[77] S. A. Mabud and A. M. Glazer, J. Appl. Crystallogr. 12, 49 (1979).

[78] G. Catalan, A. Janssens, G. Rispens, S. Csiszar, O. Seeck, G. Rijnders, D. H. A. Blank, and B. Noheda, Phys. Rev. Lett. 96, 127602 (2006).

[79] H. Morioka, T. Yamada, A. K. Tagantsev, R. Ikariyama, T. Nagasaki, T. Kurosawa, and H. Funakubo, Appl. Phys. Lett. 106, 042905 (2015).

[80] B. Noheda, D. E. Cox, G. Shirane, J. A. Gonzalo, L. E. Cross, and S.-E. Park, Appl. Phys. Lett. 74, 2059 (1999).

[81] B. Noheda, D. E. Cox, G. Shirane, J. Gao, and Z.-G. Ye, Phys. Rev. B 66, 054104 (2002).

[82] R. V. K. Mangalam, J. Karthik, A. R. Damodaran, J. C. Agar, and L. W. Martin, Adv. Mater. 25, 1761 (2013).

[83] G. Catalan, A. Lubk, A. H. G. Vlooswijk, E. Snoeck, C. Magen, A. Janssens, G. Rispens, G. Rijnders, D. H. A. Blank, and B. Noheda, Nat. Mater. 10, 963 (2011).

[84] To facilitate the comparison between the different crystallographic phases, we chose to analyze the band character by projecting onto $\mathrm{d}_{y z+z x}$ and $\mathrm{d}_{y z-z x}$ orbitals rather than the usual $d_{x z}$ and $d_{y z}$ set of orbitals. For any choice, the bands at the $\Gamma$ point are fully hybridized and these characters cannot be separated for the $P 4 \mathrm{~mm}$ phase. On the contrary, as will be clearer later, for the $\mathrm{Cm}$ and $\mathrm{Amm} 2$ structures, our choice allows us to distinguish two bands with a different character and it facilitates the discussion regarding the electric polarization's orientation.

[85] T. Wolfram and S. Ellialtioglu, Electronic and Optical Properties of $d$-Band Perovskites (Cambridge University Press, Cambridge, 2006), Chap. 10, Distorted perovskites, pp. 231248.

[86] D. C. Vaz, P. Noël, A. Johansson, B. Göbel, F. Y. Bruno, G. Singh, S. McKeown-Walker, F. Trier, L. M. Vicente-Arche, A. Sander, S. Valencia, P. Bruneel, M. Vivek, M. Gabay, N. Bergeal, F. Baumberger, H. Okuno, A. Barthélémy, A. Fert, L. Vila, I. Mertig, J.-P. Attané, and M. Bibes, Nat. Mater. 18, 1187 (2019).

[87] M. S. Bahramy, R. Arita, and N. Nagaosa, Phys. Rev. B 84, 041202(R) (2011). 